\title{
Anyon computers with smaller groups
}

\author{
Carlos Mochon* \\ Institute for Quantum Information, California Institute of Technology, Pasadena, California 91125, USA
}

(Received 10 July 2003; published 11 March 2004)

\begin{abstract}
Anyons obtained from a finite gauge theory have a computational power that depends on the symmetry group. The relationship between group structure and computational power is discussed in this paper. In particular, it is shown that anyons based on finite groups that are solvable but not nilpotent are capable of universal quantum computation. This extends previously published results to groups that are smaller and therefore more practical. Additionally, a new universal gate set is built out of an operation called a probabilistic projection, and a quasiuniversal leakage correction scheme is discussed.
\end{abstract}

DOI: 10.1103/PhysRevA.69.032306

PACS number(s): 03.67.Lx, 05.30.Pr

\section{INTRODUCTION}

The two main obstacles to building a practical quantum computer are the decoherence produced by the environment and the need for unitary operators of sufficiently high precision. Topological quantum computation provides a way of encoding quantum information in nonlocal observables that are protected from the environment, thereby solving the first problem. In some instances, the second problem can also be addressed by using operations that only depend on topological invariants.

Anyons present a concrete realization of the ideas of topological quantum computation that may have practical implementations. An anyon is a particle that has exotic quantum statistics and exists in a two-dimensional space. Anyons carry certain topological charges which cannot be locally measured or modified and can therefore be used to store protected quantum information. The charges can be detected, though, using two elementary operations called braiding and fusion. In the first operation, the positions of two anyons in the plane are exchanged, causing their world lines to braid. Because clockwise and counterclockwise rotations can be distinguished in two dimensions, braiding can produce results more complicated than the usual bosonic and fermionic cases. The second operation involves fusing two anyons into a single anyon that carries the combined charges of the original particles. In both cases, the results only depend on the charges and topological class of the paths involved. These operations can be used as a basis of error-free gates that manipulate the stored information.

Many different models of anyons can be constructed by specifying different spectra of charges together with a set of braiding and fusion rules. A convenient and physically inspired set of models can be obtained from the electric and magnetic charges of a two-dimensional finite-group gauge theory. These models depend on a finite group $G$, which acts as the symmetry of the gauge theory. While every finite group produces a consistent model, the computational power of the resulting anyons depends on the structure of the group.

Previous work by the same author [1] has shown that finite nonsolvable groups produce anyons capable of univer-

*Electronic address: carlosm@theory.caltech.edu sal quantum computation. However, the smallest finite nonsolvable group is $A_{5}$, the even permutations of five objects, which has 60 elements. Unfortunately, anyons with a large symmetry group are less likely to be found in nature and are also harder to engineer. A more desirable symmetry group would be $S_{3}$, with only 6 elements. The purpose of this paper is to study the feasibility of quantum computation with these smaller groups. In fact, it will be shown that the groups that are solvable but not nilpotent, which includes $S_{3}$ as the smallest case, produce anyons capable of universal quantum computation. The caveat, though, is that the constructions in this paper require both electric and magnetic charges, whereas magnetic charges alone were sufficient in the nonsolvable case [1]. The use of electric charges complicates the procedure significantly and will occupy the bulk of the discussion.

The ideas of this paper and its predecessor [1] are built on the foundations laid out by Kitaev [2], who introduced the notion of a quantum computer based on anyons. The first concrete description for the group $A_{5}$ was done by Ogburn and Preskill in Refs. [3,4]. An unpublished construction for the group $S_{3}$ was also worked out by Kitaev, and its use of electric charges served as a basis for much of the present work.

The organization of this paper is as follows: Section II contains a review of the basic properties of anyons and describes the notation used in this paper. The next two sections prove the universality of anyons based on groups that are semidirect products of certain cyclic groups of prime order, which includes the important case of $S_{3}$. Section III constructs an abstract set of gates out of the fundamental anyon operations, whereas Sec. IV proves that this gate set is universal. In Sec. V, the discussion is expanded to general finite groups, and the relationship between group structure and computational power is established. This section will also review the definitions of solvability and nilpotency. The main result of this paper, which is the feasibility of universal quantum computation with anyons from groups that are solvable but non-nilpotent, is proved in Sec. VI. The discussion in Sec. VI is motivated by Sec. III and includes many of the same steps, but the details are significantly more complicated. Finally, Sec. VII discusses a leakage correction scheme that can be applied to anyons, as well as many other quantum systems. 


\section{REVIEW AND NOTATION}

In this section we present a brief review of the anyon properties and operations that will be used throughout the paper. Notation for anyon pairs, qudit bases, and a special type of gate will be introduced.

The gauge theory model for anyons that is used in this paper was first presented in Ref. [5] and is summarized in the review in Ref. [6]. Our notation will be closer to the one used in the author's previous work [1] though. In the interest of space, we present only a very brief review of the basic ideas and refer the reader to the above references for further details. The only new ideas introduced in this section beyond Ref. [1] are the notation for electric charge pairs and a gate called the probabilistic projection.

\section{A. Anyon model and spectrum}

To fully characterize a system with anyons, we must specify a set of braiding and fusion rules. A set of consistent rules can be obtained from the behavior of electric and magnetic charges in a finite-group gauge theory. Though many other anyon models exist, including models with continuous groups and models with a finite spectrum that cannot be obtained from a gauge theory, only the finite-group gaugetheory model will be discussed in this paper.

For each finite group $G$, there is a gauge theory with symmetry group $G$ that contains anyons. The anyonic spectrum of the finite-group gauge theory consists of electric charges, magnetic charges, and particles called dyons which carry both electric and magnetic charge. The magnetic charges, also known as fluxes, are labeled by elements of the group $G$. The electric charges are labeled by an irreducible unitary representation $R$ of $G$ and have an internal state that transforms as a vector under $R$. The dyons are labeled by an element of $g \in G$ and a representation of the stabilizer of $g$. The dyons, however, will not play an significant role in this paper.

\section{B. Magnetic charge pairs}

We begin by discussing the braiding rules for the magnetic charges, which will be the most important particles in this paper. The basic rule for magnetic charges is the following: when two fluxes are exchanged, the flux of one is conjugated by the flux of the other. Though this is the basic interaction that will be used between magnetic charges, it has the undesirable consequence that moving single fluxes through the system can introduce unwanted correlations. Therefore, it will be necessary to work with pairs of fluxes of trivial total flux.

For any $g \in G$, we define the state $|g\rangle$ to denote a magnetic charge pair, where the first anyon has flux $g$ and the second anyon has flux $g^{-1}$. Because the effects of actions on the compensating flux $g^{-1}$ will mimic the effects on the flux $g$ we will generally not mention them explicitly. In fact, we shall refer to the state $|g\rangle$ as "a state of flux $g$," by which we describe the flux of the first anyon, rather than the total flux of the anyon pair which shall always be trivial.

A general state of $n$ magnetic charge pairs has the form

$$
|\Psi\rangle=\sum_{g_{1}, \ldots, g_{n} \in G} \alpha_{g_{1}, \ldots, g_{n}}\left|g_{1}\right\rangle \otimes \cdots \otimes\left|g_{n}\right\rangle
$$

where $\alpha_{g_{1}}, \ldots, g_{n}$ are the complex quantum amplitudes. Due to the existence of superselection sectors, which will be discussed below, the $g_{i}$ in the above sums are restricted to a single conjugacy class, which may be different for each $i$.

Because we are dealing with pairs of trivial total flux, any two states can be swapped simply by exchanging the physical position of the anyons:

$$
\sum_{g_{1}, g_{2} \in G} \alpha_{g_{1}, g_{2}}\left|g_{1}\right\rangle \otimes\left|g_{2}\right\rangle \rightarrow \sum_{g_{1}, g_{2} \in G} \alpha_{g_{1}, g_{2}}\left|g_{2}\right\rangle \otimes\left|g_{1}\right\rangle
$$

By repeatedly exchanging pairs of adjacent anyons, any two pairs of anyons can be exchanged. This operation will also allow us to move pre-made ancillas into the computational space and to move anyons that have been fused out of the computational space.

The basic interaction between pairs is a pass-through operation by which one pair passes in between a second pair. The result of the operation leaves the first pair invariant, but conjugates the second pair by either the flux of the first pair,

$$
\sum_{g_{1}, g_{2} \in G} \alpha_{g_{1}, g_{2}}\left|g_{1}\right\rangle \otimes\left|g_{2}\right\rangle \rightarrow \sum_{g_{1}, g_{2} \in G} \alpha_{g_{1}, g_{2}}\left|g_{1}\right\rangle \otimes\left|g_{1} g_{2} g_{1}^{-1}\right\rangle,
$$

or its inverse,

$$
\sum_{g_{1}, g_{2} \in G} \alpha_{g_{1}, g_{2}}\left|g_{1}\right\rangle \otimes\left|g_{2}\right\rangle \rightarrow \sum_{g_{1}, g_{2} \in G} \alpha_{g_{1}, g_{2}}\left|g_{1}\right\rangle \otimes\left|g_{1}^{-1} g_{2} g_{1}\right\rangle,
$$

depending on the direction of the pass-though. By using the swap operation, the pass-though can be performed on any two pairs of anyons.

Furthermore, the above operation can be generalized to a conjugation by a function of the fluxes of a set of anyons. That is, consider a function $f: G^{n} \rightarrow G$ that can be written as a product of its inputs, their inverses, and fixed elements of $G$. For example,

$$
f\left(g_{1}, g_{2}\right)=c_{1} g_{1}^{-1} c_{2} g_{1} c_{3} g_{1} c_{4} g_{2} c_{5},
$$

where the $\left\{c_{i}\right\}$ are fixed elements of $G$ and this case has $n$ $=2$. Then, if we assume the existence of ancillas of the form $|g\rangle$ for each $g \in G$, we can perform the unitary transformation

$$
\left|g_{1}\right\rangle \otimes\left|g_{2}\right\rangle \otimes\left|g_{3}\right\rangle \rightarrow\left|g_{1}\right\rangle \otimes\left|g_{2}\right\rangle \otimes\left|f\left(g_{1}, g_{2}\right) g_{3} f\left(g_{1}, g_{2}\right)^{-1}\right\rangle,
$$

where we have denoted its action on basis elements and the general transformation follows by linearity. The operation is implemented by conjugating in sequence by the entries of $f$, starting from right to left, where an ancilla of known flux is used for every fixed element of $f$. In general, a conjugation 
by a function can be performed with any state as target and any number of inputs, as long as the function can be written in product form.

\section{Electric charge pairs}

In addition to pairs of magnetic charges, this paper will often deal with pairs of electric charges, where the first charge transforms under the irreducible representation $R$ and the second charge transforms under the complex conjugate representation $R^{*}$. Of course, for some representations $R^{*}$ $\simeq R$, which will not be a problem for what follows.

We introduce the bases $\left\{\left|i_{R}\right\rangle\right\}$ and $\left\{\left|j_{R^{*}}\right\rangle\right\}$ on which the representations act. The indices $i, j$ take values from 1 to $d_{R}$, the dimension of the representation. We assume that the basis vectors are compatible in the sense that

$$
\left\langle i_{R^{*}}\left|R^{*}(g)\right| j_{R^{*}}\right\rangle=\left\langle i_{R}|R(g)| j_{R}\right\rangle^{*} .
$$

The combined state of the two charges is spanned by the vectors $\left|i_{R}\right\rangle \otimes\left|j_{R} *\right\rangle$ and can be described by specifying a $d \times d$ matrix $M$ :

$$
|M\rangle_{R} \equiv \frac{1}{\sqrt{d_{R}}} \sum_{i, j} M_{i j}\left|i_{R}\right\rangle \otimes\left|j_{R^{*}}\right\rangle,
$$

where we have introduced a convenient normalization factor.

We will be interested in the braiding and fusion properties of these states. However, when two electric charges move past each other, even when they are not in pairs, their charges remain unchanged. It is only the magnetic fluxes that have an effect on the electric charges. In particular, when a magnetic flux $g$ goes around an electric charge, the flux remains invariant, but the charge transforms as if multiplied by $g$ in the representation $R$. Starting with a state $|M\rangle_{R}$, if the flux circles the first electric charge, then it becomes

$$
\begin{aligned}
U(g) \otimes I|M\rangle_{R} & =\frac{1}{\sqrt{d_{R}}} \sum_{i, j, k} R_{i k}(g) M_{k j}\left|i_{R}\right\rangle \otimes\left|j_{R^{*}}\right\rangle \\
& =|R(g) M\rangle_{R},
\end{aligned}
$$

where $R(g) M$ is the matrix obtained by left multiplying $M$ by the element $g$ in the representation $R$. Similarly, if we act on the second charge, we obtain

$$
\begin{aligned}
I \otimes U(g)|M\rangle_{R} & =\frac{1}{\sqrt{d_{R}}} \sum_{i, j, k} M_{i k} R_{j k}^{*}(g)\left|i_{R}\right\rangle \otimes\left|j_{R^{*}}\right\rangle \\
& =\frac{1}{\sqrt{d_{R}}} \sum_{i, j, k} M_{i k} R_{k j}^{\dagger}(g)\left|i_{R}\right\rangle \otimes\left|j_{R^{*}}\right\rangle \\
& =\left|M R\left(g^{-1}\right)\right\rangle_{R},
\end{aligned}
$$

where we have used the fact that $R$ is unitary.

Note that, just as in the case of the magnetic charges, if we have a function $f\left(\left\{g_{i}\right\}\right)$ of some anyon fluxes, written out in product form, then we can apply this function to our charges,

$$
|M\rangle_{R} \rightarrow U(f) \otimes I|M\rangle_{R}=|R(f) M\rangle_{R},
$$

by applying sequentially from right to left the elements of the product.

\section{Superselection sectors, fusion, and vacuum pairs}

Before describing the fusion rules for the magnetic and electric charges, we need to address the issue of superselection sectors, which is familiar to particle physicists. A superselection sector is a subspace of a Hilbert space that is invariant under all the implementable transformations. A useful analogy is to consider the Hilbert space of a particle called the nucleon, spanned by the four states

$$
|0 \uparrow\rangle,|0 \downarrow\rangle,|1 \uparrow\rangle,|1 \downarrow\rangle,
$$

corresponding to a spin- $-\frac{1}{2}$ particle with two possible charge values. This is nothing more than the direct sum of the Hilbert spaces of the proton and neutron:

$$
\mathcal{H}_{\text {nucleon }}=\mathcal{H}_{\text {proton }} \oplus \mathcal{H}_{\text {neutron }} .
$$

At the energies of atomic physics, it is not possible to measure in the proton plus neutron basis or to perform a unitary rotation along this direction. Therefore, we could say that a nucleon automatically decoheres into either a proton or a neutron.

A similar situation occurs with the anyons. Each conjugacy class of $G$ is a magnetic charge superselection sector. The irreducible representations are the electric charge superselection sectors. When given an unknown anyon-for example, an anyon created from the vacuum-we can assume that it has decohered into a specific, though possibly unknown, conjugacy class and/or irreducible representation. Furthermore, when storing quantum information, it will be important to keep the computational space in a single superselection sector to avoid decoherence.

Let $|\Psi\rangle$ be a pair of anyons created from the vacuum. We may assume that each anyon has decohered into a specific superselection sector. Furthermore, because a vacuum pair must consist of a particle with its antiparticle, the two superselection sectors are related. That is, the pair must have vacuum quantum numbers and be able to fuse back into the vacuum. Therefore, if the first anyon is a magnetic charge with flux in a given conjugacy class, the second anyon will be a magnetic charge with flux in the inverse conjugacy class. If the first anyon is an electric charge of representation $R$, then the second anyon will be a electric charge of the complex conjugate representation. Finally, if one anyon is a dyon, then so is the other.

In the case of magnetic charges, there is exactly one state with vacuum quantum numbers in each conjugacy class. The state is

$$
|\operatorname{Vac}(\mathcal{C})\rangle=\frac{1}{\sqrt{|\mathcal{C}|}} \sum_{g \in \mathcal{C}}|g\rangle,
$$

where $\mathcal{C}$ is a conjugacy class of $G$. Note that, given our notation, the above state is an entangled state of two anyons. 
In the case of electric charges, the vacuum state for representation $R$ is simply $|R(I)\rangle_{R}$, where $R(I)$ is the $d_{R} \times d_{R}$ identity matrix.

The operation of fusion is in a sense the inverse of vacuum pair creation. Fusing two anyons produces a single anyon that must carry the total magnetic and electric charges of the pair. In the special case when both total charges are trivial (i.e., one of the above vacuum states) the state can fuse into the vacuum, leaving no particle behind and transferring its energy to some other medium such as photons. In theory, this case can easily be detected in the laboratory and is the primary way of obtaining measurement results.

In the case of magnetic charges, the net resulting flux is just the product of the two fluxes, where the ordering of the product depends on some conventions which will not be important here. While one of our standard anyon pairs always has trivial total flux, we sometimes may fuse anyons from different pairs to determine if their flux is equal. Even if the total flux is trivial, though, the pair may not fuse into the vacuum but may produce an electric charge. This will be the case if the state transforms nontrivially under simultaneous conjugation of both anyons.

The fusion of two electric charges can only produce another electric charge (or the vacuum, which is the charge carrying the trivial representation). To calculate the possible products of fusion, note that fusion implies that a flux can no longer be braided around only one of the two electric charges. Mathematically, it is a restriction to the diagonal transformations

$$
|M\rangle_{R} \rightarrow U(g) \otimes U(g)|M\rangle_{R}=\left|R(g) M R\left(g^{-1}\right)\right\rangle_{R} .
$$

However, the above action of the group is not irreducible on this space. The vector space spanned by all possible states $|M\rangle_{R}$ decomposes into invariant subspaces. The invariant subspaces correspond to electric charges transforming under irreducible representations. The probability of obtaining each irreducible representation corresponds to the magnitude of the state vector projected down to the appropriate invariant subspace. Furthermore, after fusion, it is no longer possible to measure the relative phase between the different representations and therefore decoherence occurs in the representation basis.

The net result of fusion is a mixed state of different representations. Which representations occur is determined by the decomposition of $R(g) \otimes R^{*}(g)$ into irreducible representations. The probability of obtaining each of these representations is determined by the projection of $M$ to the different invariant subspaces.

In particular, the trace of $M$ is the unique invariant under conjugation by $G$ (which is the content of Schur's lemma). Therefore the probability of fusion into the vacuum is

$$
P_{\mathrm{vac}}=\left|\langle R(1) \mid M\rangle_{R}\right|^{2}=\left|\frac{\operatorname{Tr}(M)}{d_{R}}\right|^{2} .
$$

\section{E. Requirements for the physical system}

To complete our review of the properties of anyons, we will list the operations, ancillas, and measurements that we assume are available on any realistic system and which we will use to build our quantum gate set:

(1) We can braid or exchange any two particles.

(2) We can fuse a pair of anyons and detect whether there is a particle left behind or whether they had vacuum quantum numbers.

(3) We can produce a pair of anyons in a state that is chosen at random from the two-particle subspace that has vacuum quantum numbers.

(4) We have a supply of ancillas of the form $|g\rangle$ for any $g \in G$.

(5) We have a supply of ancillas of the form $|R(I)\rangle_{R}$ for any irreducible unitary representation $R$.

The last two requirements are the only questionable ones, as it is not obvious how to produce this reservoir of calibrated electric and magnetic charges. In fact, since many of these ancillas will be destroyed during fusion, the reservoir will have to have a large number of ancillas of each type.

One of the main difference between the constructions in this paper and the one used in producing computations with nonsolvable groups [1] is that the latter case required no electric charge ancillas, which may be harder to produce. Additionally, Ref. [1] presented a protocol for producing the magnetic ancillas for a simple non-Abelian group. The production of calibrated flux and charge ancillas for the groups discussed in the present paper, though similar, will not be addressed here.

A final note is that the requirement of calibrated magnetic charge ancillas will have to be slightly modified in Sec. VI C, in order to work with certain large groups.

\section{F. Notation for qudits}

Throughout this paper it will be useful to perform computations with qudits rather than the usual qubits. We define our computational basis as the states $|i\rangle$ for $0 \leqslant i<d$, where we will assume that $d$ is prime. The unitary $Z$ and $X$ gates can be defined as follows:

$$
\begin{gathered}
Z|i\rangle=\omega^{i}|i\rangle, \\
X|i\rangle=|i+1\rangle,
\end{gathered}
$$

where $\omega$ is a fixed nontrivial $d$ th root of unity and sums are understood to be modulo $d$. As usual, the eigenstates of $Z$ correspond to the computational basis. We can also introduce the eigenstates of $X$,

$$
|\tilde{i}\rangle=\frac{1}{\sqrt{d}} \sum_{j=0}^{d-1} \omega^{-i j}|j\rangle,
$$

which have the following transformations under the action of our unitary gates:

$$
\begin{aligned}
& Z|\widetilde{i}\rangle=|\widetilde{i-1}\rangle, \\
& X|\widetilde{i}\rangle=\omega^{i}|\widetilde{i}\rangle .
\end{aligned}
$$

Note that when appropriate, we shall assume all operations are modulo $d$ without further comment. 


\section{G. Probabilistic projection onto $\mathcal{K}$}

To conclude with the introduction of notation, we define a new type of gate called a probabilistic projection onto a subspace. The operation is essentially a projective measurement that distinguishes between a subspace $\mathcal{K}$ and its orthogonal complement. However, the operation has a one-sided probability of error, corresponding to a failure to notice the projection into $\mathcal{K}$.

For example, consider an operation that emits a photon if and only if the state is projected into the subspace $\mathcal{K}$. The photon is then received at a photodetector that has a probability $0<p \leqslant 1$ of absorbing the photon. A photon will never be detected if the state was projected into the complement of $\mathcal{K}$, but even if the measurement projected into $\mathcal{K}$, the photodetector may remain silent.

To formalize the idea of a probabilistic projection, let $\mathcal{K}$ be a subspace of a Hilbert space $\mathcal{H}$ and let $P_{\mathcal{K}}$ be the projection onto $\mathcal{K}$. We define a probabilistic projection onto $\mathcal{K}$ as a two-outcome POVM with operators

$$
F_{0}=p_{P P} P_{\mathcal{K}}, \quad F_{1}=1-p_{P P} P_{\mathcal{K}}
$$

where $0<p_{P P} \leqslant 1$. We say that we can do a probabilistic projection onto $\mathcal{K}$ if we can do the above operation for any fixed $p_{P P}$.

Furthermore, we demand that if outcome 0 is obtained when applying the operation to a state $|\Psi\rangle$, we obtain the state

$$
\left|\Psi_{0}\right\rangle=\frac{P_{\mathcal{K}}|\Psi\rangle}{\sqrt{\left\langle\Psi\left|P_{\mathcal{K}}\right| \Psi\right\rangle}} .
$$

On the other hand, if we get the result 1 , we will consider the state damaged, and trace it out of our computational system.

As an example consider

$$
|\Psi\rangle=\frac{1}{\sqrt{2}}(|0\rangle \otimes|1\rangle+|1\rangle \otimes|0\rangle)
$$

and let $\mathcal{K}=\{|0\rangle\}$. Applying a probabilistic projection to the first qubit, we obtain with probability $p_{P P} / 2$ the state

$$
\left|\Psi_{0}\right\rangle=|0\rangle \otimes|1\rangle
$$

and with probability $1-p_{P P} / 2$ we obtain the mixed state

$$
\rho_{1}=\frac{1}{2-p_{P P}}\left[\left(1-p_{P P}\right)|1\rangle\langle 1|+| 0\rangle\langle 0|\right],
$$

where we have already traced out the first qubit. Notice that if the probabilistic projection onto $|0\rangle$ is applied to both qubits simultaneously, it is possible to obtain the result 1 twice, but it is not possible to obtain the result 0 twice.

\section{BASE CASE: $G=\mathbb{Z}_{P} \times_{\theta} \mathbb{Z}_{Q}$}

Before tackling the general case of groups that are solvable but not nilpotent, we will describe the procedure for producing quantum computation using a special type of group based on the semidirect product. The construction for these groups is very similar to the general case, but can be described in more concrete terms. In particular, these groups are very useful in eliminating operations whose usefulness is unclear in the general case, but that have no computational power when reduced to this special case.

\section{A. Algebraic structure}

We will be interested in the groups $G=\mathbb{Z}_{p} \times{ }_{\theta} \mathbb{Z}_{q}$, the semidirect product of the cyclic groups of order $p$ and $q$. We assume that $p \neq q$ are both prime and that the function $\theta$ is nontrivial, which guarantees that $G$ is not nilpotent.

The group can be described using two generators $a$ and $b$ which satisfy the relations

$$
a^{p}=1, \quad b^{q}=1, \quad b a b^{-1}=a^{t},
$$

where specifying an integer $t$ between 0 and $p$ is equivalent to specifying the function $\theta: \mathbb{Z}_{q} \rightarrow \operatorname{Aut}\left(\mathbb{Z}_{p}\right)$ used for the semidirect product. We will require that $t \neq 1$ which is equivalent to $\theta$ being nontrivial. Furthermore, consistency requires that

$$
a=b^{q} a b^{-q}=a^{t^{q}} \Rightarrow t^{q}=1 \bmod p,
$$

which can always be solved for some $t$ as long as $q$ divides $p-1$. We henceforth assume that $p, q$, and $t$ have been chosen in a self-consistent fashion.

The best example of one of these groups and, in fact, the smallest non-Abelian group is $S_{3}$. This group can be expressed as $\mathbb{Z}_{3} \times_{\theta} \mathbb{Z}_{2}$, with $t=2$. We can choose $a$ to be any order three element such as (123), and we can choose $b$ to be any order two element such as (12).

The first example of such a group with odd order is $\mathbb{Z}_{7}$ $\times_{\theta} \mathbb{Z}_{3}$ with $t=2$ or $t=4$, both of which are equivalent. One of the most important features of this example is that not all the nontrivial powers of $a$ are conjugate to one another. The elements $a, a^{2}$, and $a^{4}$ form one conjugacy class, whereas the elements $a^{3}, a^{5}$, and $a^{6}$ form another.

Both of the above examples will be revisited when we discuss group representations and fusion of electric charges.

\section{B. Computational basis}

We choose a qudit computational basis

$$
|i\rangle=\left|a^{i} b a^{-i}\right\rangle
$$

for $0 \leqslant i<p$. Note that all these states are unique because $a^{i} b a^{-i}=a^{i(1-t)} b$ and $a^{1-t}$ is a nontrivial generator of the group $\mathbb{Z}_{p}$. We are therefore using a complete conjugacy class for the computational subspace.

While the above choice of computational subspace may seem arbitrary, most other choices are either equivalent or less powerful. The conjugacy classes $a^{i} b^{j} a^{-i}$, for different nontrivial values of $j$, are all equivalent. Dyons with these fluxes are also equivalent since they are just the combination of the above states with electric charges that cannot be detected by braiding. Finally, the powers of $a$ and pure electric 
charges are suboptimal as they are difficult to entangle (for more on this see the discussion on using nilpotent groups in Sec. V).

Initializing a quantum computer in this basis is easy, as we have assumed the existence of flux ancillas in the state $|0\rangle$, which can be used as computational anyons. We therefore turn to the task of implementing gates on this space.

\section{Operations involving braiding fluxes}

We begin by characterizing the operations that can be achieved by braiding fluxes. Fix a target qudit which we will be conjugating, and assume that it is in the computational subspace. We can conjugate this qudit by the fluxes of arbitrary ancillas in the group. It can also be conjugated by the fluxes of other qudits, which we will also assume to have a definite flux in the computational subspace (as the effect of a superposition of fluxes can be inferred by linearity).

Let us begin with the case when only one qudit (in addition to the target) is involved. If the source qudit is in a state $|g\rangle$, then the target will get conjugated by an expression

$$
f(g)=c_{1} g c_{2} g c_{3} \cdots c_{n}
$$

for some $n$, where the $\left\{c_{j}\right\}$ are fixed elements of $G$ corresponding to the ancillas used. Of course, these elements represent the product of any ancillas that were used in series and can equal the identity if no ancillas were used.

Because of the structure of the group, all the fixed elements can be expanded as $c_{i}=a^{j_{i}} b^{k_{i}}$ for some integers $j_{i}$, $k_{i}$. Furthermore, since the source flux is in the computational basis, it can be written out as $g=a^{x} b a^{-x}=a^{x(1-t)} b$ for some $x$. Inserting these expressions, we get

$$
f(g)=a^{j_{1}} b^{k_{1}} a^{x(1-t)} b a^{j_{2}} b^{k_{2}} \cdots a^{j_{n}} b^{k_{n}} .
$$

Using the group relation $b a^{i}=a^{i t} b$, we can move all the $b$ 's to the right and combine factors to get

$$
f(g)=a^{\alpha} a^{\beta x} b^{\delta}
$$

for some integers $\alpha, \beta$, and $\delta$. The effect of each of these factors can be considered separately. Conjugating by $a^{\alpha}$ is just the application of the gate $X^{\alpha}$. Conjugating by $a^{\beta x}$ is just a controlled- $X$ gate from the source to the target, repeated $\beta$ times. Finally, conjugating by $b$ maps $|i\rangle$ to $|i t\rangle$. This operation can be generated using a controlled- $X$ gate and an ancilla $|0\rangle$ :

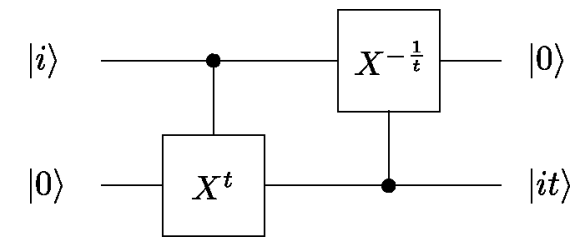

where $-1 / t$ is computed modulo $p$. Following the above circuit, we can either replace the original qudit with the ancilla or use a swap, which can also be built out of controlled$X$ gates.
So far we have shown that the $X$ and controlled- $X$ gates generate the set of operations achieved by conjugations. However, we have yet to show that these operations are in fact included in the set of achievable operations. The $X$ gate is rather trivial as it is a conjugation by an ancilla of flux $a$. The controlled- $X$ gate is a conjugation by the function

$$
f(g)=\left(g b^{-1}\right)^{1 /(1-t) \bmod p}=\left(a^{x(1-t)} b b^{-1}\right)^{1 /(1-t) \bmod p}=a^{x},
$$

where $1 /(1-t)$ can be computed modulo $p$ because we assumed $1<t<p$.

The case involving many source qudits, all of which can be used to conjugate the target, is very similar to the above. The expression can be simplified by moving all the $b$ 's to the left and combining similar factors. In the end, the net effect will again be a series of $X$ and controlled- $X$ gates.

Finally, one may wonder about using an ancilla as an intermediate step. That is, first we take an ancilla (say, $g^{\prime}$ ), conjugate it by some function (say, $f$ ) of some qudits, and then conjugate the target by the ancilla. However, the same effect can be achieved by conjugating the target first by $f^{-1}$, then by $g^{\prime}$, and finally by $f$. This procedure therefore provides no extra computational power.

The conclusion is that the operations achievable from braiding magnetic charges are exactly those generated by the $X$ and controlled- $X$ gates. In fact, the $X$ gate is redundant as we have assumed the existence of $|1\rangle$ ancillas, which can be used as control qudits in a controlled- $X$ gate.

\section{Operations involving fusion of fluxes}

Now we turn to the operations achieved by the fusion of magnetic fluxes. For these operations it will be sufficient to determine whether the two particles fused into the vacuum or not, thereby obtaining at most one bit of information from each fusion.

At this point we remind the reader that standard states consist of pairs of anyons, whose total flux is trivial. That is, the state $|g\rangle$ describes an anyon of flux $g$ paired with an anyon of flux $g^{-1}$. There are therefore two basic choices for fusion: we can fuse the two anyons that compose a single pair with each other or we can fuse one of them with an anyon from another pair, typically an ancilla. To avoid confusion, in the latter case we will always use the anyon of flux $g$ (rather than $g^{-1}$ ) for the fusion.

The case of fusion with an ancilla will lead to a measurement in the $Z$ basis. The fusion of anyons from the same pair will lead to a measurement in the $X$ basis. However, we will delay the construction of the actual measurement gates until the next section. For this section, we will simply describe the fusions as abstract operations on the computational space by employing the construction of probabilistic projections.

The fusion of an anyon from a state $|\Psi\rangle$ with an anyon ancilla of flux $b^{-1}$ is a probabilistic projection onto the subspace $\mathcal{K}=\{|0\rangle\}$. That is, an anyon of flux $a^{i} b a^{-i}$ can only fuse into the vacuum with a flux $b^{-1}$ if $i=0$ (modulo $p$ as usual). When $i>0$ there must be an anyon left over to carry the nontrivial total flux. When $i=0$ the fusion can either produce the vacuum state or an anyon with nontrivial charge. 
The probability for fusion into the vacuum in this case is $1 / p$. Furthermore, if we fuse into the vacuum we can replace the state with a $|0\rangle$ ancilla. Therefore the whole operation is a probabilistic projection onto $|0\rangle$ with $p_{P P}=1 / p$.

The fusion of two anyons from the same pair is a probabilistic projection onto the subspace $\mathcal{K}=\{|\widetilde{0}\rangle\}$. Because the total magnetic flux of the pair is always trivial, the fusion product must be an electric charge. The charge corresponds to a representation of $G$ given by the action of conjugation on the anyon fluxes. The state $|\widetilde{0}\rangle$ transforms trivially and corresponds to the vacuum, whereas the states $|\widetilde{i}\rangle$, for $i$ $>0$, are orthogonal to the vacuum and correspond to nontrivial representations. In fact, this procedure is a probabilistic projection with $p_{P P}=1$. However, since the state is destroyed during fusion, to complete the projection we must be able to produce $|\widetilde{0}\rangle$ states. This will be discussed below.

The other choices for fusion are equivalent to a combination of one of the above measurements and an $X$ or controlled- $X$ gate. Fusing with a flux of the form $a^{i} b^{-1} a^{-i}$ is equivalent to first applying a $X^{-i}$ gate and then performing a fusion with $b^{-1}$. A fusion with any other flux can never produce the vacuum if the qudit is in the computational subspace. Finally, one can consider fusion of anyons from two different qudits. If the state of the two qudits is $|i\rangle \otimes|j\rangle$, the fusion will only produce the vacuum state if $i=j$. Therefore, the operation can be simulated by a controlled- $X^{-1}$ gate, followed by the fusion of the target with a $b^{-1}$ flux.

The conclusion so far is that fusion of magnetic charges provides us with two new operations: the probabilistic projections onto the subspaces $|0\rangle$ and $|\widetilde{0}\rangle$, which will eventually become measurements in the $Z$ and $X$ bases. The only operation that has not been considered is using the products of fusion for further operations or fusions. This subject will be briefly touched upon after discussing fusion of electric charges.

\section{Production of $|\widetilde{\mathbf{0}}\rangle$ states}

To conclude the discussion on fusion of fluxes, we present the construction of $|\widetilde{0}\rangle$ states, which were needed to complete the probabilistic projection onto $|\widetilde{0}\rangle$.

Just as the state $|\widetilde{0}\rangle$ naturally fuses into the vacuum, it is also naturally produced from the vacuum. Unfortunately, producing a pair of anyons from the vacuum is just as likely to produce the vacuum state for one of the other superselection sectors as it is to produce the state $|\widetilde{0}\rangle$. Therefore, after producing a vacuum state we must measure its superselection sector. Vacuum pairs that are produced in the computation subspace (magnetic charge in the conjugacy class of $b$ ) will be kept as $|\widetilde{0}\rangle$ states and the rest will be tossed out.

Since measurements are done by fusion, which is a destructive procedure, we must copy the vacuum state before measuring the conjugacy class. The procedure starts with a pair created from the vacuum and a $|0\rangle$ ancilla,

$$
|\mathrm{Vac}\rangle \otimes|0\rangle
$$

and applies to it a swap, made out of the conjugation-based controlled $X$ :

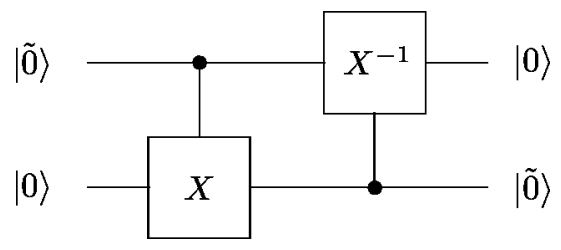

where the circuit depicts the result for the case when the vacuum pair was created in the computational superselection sector, in which case $|\mathrm{Vac}\rangle=|\widetilde{0}\rangle$.

In the case when the vacuum state was not created in the computational superselection sector, then the effect of the conjugations will be different. However, since the conjugations are performed using braiding, which never changes the superselection sector, the vacuum state can only be transformed into a state that is orthogonal to $|0\rangle=|b\rangle$.

After applying the above controlled- $X$ gates, we attempt to fuse an anyon from what was the vacuum state with an ancilla of flux $b^{-1}$. If they fuse into the vacuum, this implies that the vacuum state was created in the computational superselection sector and the above circuit worked correctly. The ancillas $|0\rangle$ will have been transformed properly into a $|\widetilde{0}\rangle$ ancilla, which can be used for computation. In the case when the fusion does not produce a vacuum state, the swap probably did not produced the desired state, so we discard it and start over.

To summarize, we now have a source of $|\widetilde{0}\rangle$ ancillas, which can be used as the last step needed to complete the probabilistic projection onto $|\widetilde{0}\rangle$.

\section{E. Representations and fusion of electric charges}

Thus far, we have only considered operations involving magnetic fluxes. These operations led to a controlled- $X$ gate and measurements in the $X$ and $Z$ bases. However, these gates do not form a universal gate set. We must therefore consider operations involving electric charges as well.

The electric charges transform as irreducible representation of the group $G$. To obtain the spectrum of electric charges, as well as their braiding and fusion rules, we must therefore discuss the representation theory of $G$.

It is easy to see that the commutator subgroup $G^{\prime}$ of groups of the form $G=\mathbb{Z}_{p} \times_{\theta} \mathbb{Z}_{q}$ is just $G^{\prime}=\mathbb{Z}_{p}$. The representation theory of $G$ can be obtained by inducing representations from $G^{\prime}$. Starting from the trivial representation on $G^{\prime}$, the induced representations are the one dimensional representations where $a \rightarrow 1$ and $b$ is a $q$ th root of unity.

The rest of the irreducible representations have dimension $q$ and are obtained by inducing from the nontrivial representations of $\mathbb{Z}_{p}$. The induced representations are all irreducible though not necessarily distinct. In fact, they can be easily described in their natural basis as 


$$
a \rightarrow\left(\begin{array}{cccc}
\omega & & & \\
& \omega^{t} & & \\
& & \ddots & \\
& & & \omega^{t^{q-1}}
\end{array}\right), \quad b \rightarrow\left(\begin{array}{cccc}
0 & 1 & & \\
& 0 & & \\
& & \ddots & 1 \\
1 & & & 0
\end{array}\right),
$$

where $\omega$ is the $p$ th root of unity of the representation from which we are inducing. The matrix for $a$ is diagonal, whereas $b$ is the permutation matrix with entries 1 above the diagonal.

Even though the representation theory for these particular groups is easy, we will use abstract language to describe the fusion rules, which will make the connection to the general case clearer.

Take any non-Abelian irreducible representation, and consider a pair of electric charges in the state $\left|R\left(a^{i}\right)\right\rangle_{R}$. What representations do we get if we fuse the two charges? The product of fusion is invariant under the action of $a$,

$$
U(a) \otimes U(a)\left|R\left(a^{i}\right)\right\rangle_{R}=\left|R(a) R\left(a^{i}\right) R\left(a^{-1}\right)\right\rangle_{R}=\left|R\left(a^{i}\right)\right\rangle_{R},
$$

and therefore represents the commutator subgroup $G^{\prime}$ by the identity. This implies that the representation is Abelian. In particular, it is easy to see that the one-dimensional subspaces

$$
\left|\left[\gamma^{j}\right]\right\rangle_{R} \equiv\left|\operatorname{diag}\left(\gamma^{j}, \gamma^{2 j}, \ldots, \gamma^{q j}\right)\right\rangle_{R},
$$

with $\gamma^{q}=1$, are the spaces corresponding to the representations $a \rightarrow 1, b \rightarrow \gamma^{j}$.

We will be interested in the quantum amplitude that a state $\left|R\left(a^{i}\right)\right\rangle_{R}$ fuses into the $b \rightarrow \gamma^{j}$ representation. This quantity will be denoted by the fusion amplitude

$$
F_{i \rightarrow j} \equiv\left\langle\left[\gamma^{j}\right] \mid R\left(a^{i}\right)\right\rangle_{R}=\frac{1}{q} \sum_{k=1}^{q} \gamma^{-k j} \omega^{i t^{(k-1)}},
$$

with $0 \leqslant i<p$ and $0 \leqslant j<q$.

Let $|\Psi\rangle$ be an arbitrary state entangled with an electric charge pair:

$$
|\Psi\rangle=\sum_{i=0}^{p-1}\left|\Psi_{i}\right\rangle \otimes\left|R\left(a^{i}\right)\right\rangle_{R}
$$

where the $\left|\Psi_{i}\right\rangle$ denotes (unnormalized) states of the rest of the system. The fusion amplitudes allow $|\Psi\rangle$ to be rewritten as

$$
|\Psi\rangle=\sum_{i=0}^{p-1} \sum_{j=0}^{q-1} F_{i \rightarrow j}\left|\Psi_{i}\right\rangle \otimes\left|\left[\gamma^{j}\right]\right\rangle_{R}
$$

The basis $\left|\left[\gamma^{j}\right]\right\rangle_{R}$ labels the total charge of the two anyons that comprise the electric charge pair. A fusion of the two electric charges, followed by a measurement of the resulting fusion product, will be a measurement in this basis.

Note that the basis $\left|\left[\gamma^{j}\right]\right\rangle_{R}$ only spans the diagonal subspace of $|M\rangle_{R}$. However, this is the subspace containing all the states $\left|R\left(a^{i}\right)\right\rangle_{R}$. The subspaces spanned by $\left|R\left(b^{j} a^{i}\right)\right\rangle_{R}$, for some fixed $j>0$, are mapped unchanged into the space of a single higher-dimensional irreducible representation and are therefore not useful for the purposes of this paper.

While the representation $R$ does not appear explicitly in the fusion coefficients, it enters implicitly in the above expression as the choice for $p$ th root of unity $\omega$. Though we could use the notation $\omega_{R}$, this will not be necessary as we will generally work with only one higher-dimensional irreducible representation.

The most important feature of the $F_{i \rightarrow j}$ coefficients is that $\left|R\left(a^{0}\right)\right\rangle_{R}$ is the vacuum state and therefore

$$
F_{0 \rightarrow j}=\delta_{j, 0},
$$

which can be verified by direct calculation. Another important property is that

$$
\left|F_{i \rightarrow j}\right|>0
$$

for all $i>0$. The proof involves showing that a linear relation of roots of unity only vanishes if it is a combination of the obvious regular polygon relations (which is proved in [7]).

A final interesting property is that

$$
F_{i t^{k \rightarrow j}}=\gamma^{-j k} F_{i \rightarrow j},
$$

which is a consequence of

$$
\left|R\left(a^{i t^{k}}\right)\right\rangle_{R}=\left|R\left(b^{k} a^{i} b^{-k}\right)\right\rangle_{R}=U\left(b^{k}\right) \otimes U\left(b^{k}\right)\left|R\left(a^{i}\right)\right\rangle_{R} .
$$

\section{F. Examples}

\section{1. $S_{3}$}

The group $S_{3}$ has three irreducible representations, the trivial (identity) representation (where $a \rightarrow 1, b \rightarrow 1$ ), the sign of the permutation (where $a \rightarrow 1, b \rightarrow-1$ ), and a twodimensional one:

$$
a \rightarrow\left(\begin{array}{cc}
\omega & 0 \\
0 & \bar{\omega}
\end{array}\right), \quad b \rightarrow\left(\begin{array}{ll}
0 & 1 \\
1 & 0
\end{array}\right),
$$

where $\omega$ is a nontrivial cube root of unity. The fusion amplitudes are

$$
\begin{gathered}
F_{0 \rightarrow 0}=1, \quad F_{1 \rightarrow 0}=-\frac{1}{2}, \quad F_{2 \rightarrow 0}=-\frac{1}{2}, \\
F_{0 \rightarrow 1}=0, \quad F_{1 \rightarrow 1}=-i \frac{\sqrt{3}}{2}, \quad F_{2 \rightarrow 1}=i \frac{\sqrt{3}}{2} .
\end{gathered}
$$

The best way to visualize these coefficients is to start with a state $|\widetilde{0}\rangle$ and a pair of electric charges in the vacuum state of the two-dimensional representation: $|R(I)\rangle_{R}$. Then entangle with a controlled sum to get

$$
\frac{1}{\sqrt{3}} \sum_{j}|j\rangle\left|R\left(a^{j}\right)\right\rangle_{R}=\frac{1}{\sqrt{3}} \sum_{j}|j\rangle\left|\left(\begin{array}{cc}
\omega^{j} & 0 \\
0 & \bar{\omega}^{j}
\end{array}\right)\right\rangle_{R} .
$$


Fusion of the electric charge pair produces either the vacuum (trivial representation) or a charge transforming under the sign representation. The probability of getting each is

$$
\begin{aligned}
& P_{v a c}=\sum_{j}\left|\frac{1}{\sqrt{3}} F_{j \rightarrow 0}\right|^{2}=\frac{1}{2}, \\
& P_{s g n}=\sum_{j}\left|\frac{1}{\sqrt{3}} F_{j \rightarrow 1}\right|^{2}=\frac{1}{2},
\end{aligned}
$$

and the state of the magnetic charges afterwards is one of

$$
\begin{gathered}
\left|\Psi_{\text {vac }}\right\rangle=\frac{1}{\sqrt{6}}(2|0\rangle-|1\rangle-|2\rangle), \\
\left|\Psi_{\text {sgn }}\right\rangle=\frac{1}{\sqrt{2}}(|1\rangle-|2\rangle) .
\end{gathered}
$$

These are obtained by multiplying the initial state by the appropriate $F$ coefficients and renormalizing to unit magnitude. In the case of the second state we also introduced an extra global phase of $i$, which is related to the arbitrary choice of phase of the $\left|\left[\gamma^{j}\right]\right\rangle_{R}$ states.

$$
\text { 2. } Z_{7} \times_{\theta} Z_{3}
$$

The group $\mathbb{Z}_{7} \times_{\theta} \mathbb{Z}_{3}$ has five irreducible representations. Three of them are one dimensional and set $a \rightarrow 1$ and $b$ to a cube root of unity. The other two are three dimensional and are complex conjugates of each other.

The main new feature of this group is that the nontrivial powers of $a$ are not all conjugate to one another. This leads to more complicated fusion coefficients. For example,

$$
\begin{array}{ccc}
F_{0 \rightarrow 1}=0, & \\
F_{1 \rightarrow 1}=\frac{1}{3} A, & F_{2 \rightarrow 1}=\frac{\gamma^{2}}{3} A, & F_{3 \rightarrow 1}=\frac{\gamma}{3} B, \\
F_{4 \rightarrow 1}=\frac{\gamma}{3} A, & F_{5 \rightarrow 1}=\frac{\gamma^{2}}{3} B, & F_{6 \rightarrow 1}=\frac{1}{3} B,
\end{array}
$$

with

$$
\begin{gathered}
A=\gamma^{2} \omega+\gamma \omega^{2}+\omega^{4}=e^{2 \pi i(17 / 21)}+e^{2 \pi i(13 / 21)}+e^{2 \pi i(12 / 21),} \\
B=\gamma^{2} \omega^{-1}+\gamma \omega^{-2}+\omega^{-4}=e^{2 \pi i(11 / 21)}+e^{2 \pi i(1 / 21)}+e^{2 \pi i(9 / 21)},
\end{gathered}
$$

where we have chosen $\gamma=\mathrm{e}^{2 \pi i / 3}$ and $\omega=\mathrm{e}^{2 \pi i / 7}$. Notice how $A$ is close in magnitude to 3 whereas $B$ is close in magnitude to 1 .

\section{G. Operations involving electric charges}

Now it is time to apply the discussion in the previous subsections to build a useful operation out of electric charges. While there seems to be a wealth of strange ancillas that could be produced using electric charges, most of them have complicated relative amplitudes or phases that are hard to use in a constructive proof of universal computation. We will therefore focus our attention on producing an operation that arises naturally from the fusion amplitudes: the projection onto the subspace orthogonal to $|0\rangle$.

Consider a qudit in the state

$$
|\Psi\rangle=\sum_{i=0}^{p-1} \psi_{i}|i\rangle
$$

where the coefficients $\left\{\psi_{i}\right\}$ could either be complex numbers or could represent the state of the rest of the system if the qudit is entangled with other qudits.

We append to the qudit an electric charge pair $|R(I)\rangle_{R}$ in the vacuum state of a non-Abelian representation $R$. Using braiding, we can right multiply the state of the electric charge by some function $f$ of the qudits flux:

$$
|\Psi\rangle \otimes|R(I)\rangle_{R} \rightarrow \sum_{i=0}^{p-1} \psi_{i}|i\rangle \otimes|R(f(i))\rangle_{R} .
$$

We have shown in Sec. III C that the most general function is of the form $f(i)=a^{\alpha} a^{\beta i} b^{\delta}$. Choosing $\delta \neq 0$ turns out not to be useful, and choosing $\alpha \neq 0$ can be used to get projections to the spaces orthogonal to $|i\rangle$ for $i>0$, but this can be achieved as well with an $X$ gate. We will therefore focus on $f(i)=a^{\beta i}$ so that we obtain the state

$$
\sum_{i=0}^{p-1} \psi_{i}|i\rangle \otimes\left|R\left(a^{\beta i}\right)\right\rangle_{R}=\sum_{i=0}^{p-1} \sum_{j=0}^{q-1} F_{\beta i \rightarrow j} \psi_{i}|i\rangle \otimes\left|\left[\gamma^{j}\right]\right\rangle_{R} .
$$

A fusion of the electric charge pair, followed by a measurement of the resulting electric charge (the feasibility of which will be the subject of Sec. III H below), leads to a state that is proportional to

$$
\sum_{i=0}^{p-1} F_{\beta i \rightarrow j} \psi_{i}|i\rangle
$$

where $j$ now labels the result of the measurement in the basis $\left|\left[\gamma^{j}\right]\right\rangle_{R}$.

Because of the property $F_{0 \rightarrow j}=\delta_{j, 0}$, if the measurement result is $j \neq 0$, we will have projected into the space orthogonal to $|0\rangle$. Unfortunately, we will have also introduced undesired relative phases and amplitudes. The trick will be to balance these out.

Consider repeating the above procedure $p-1$ times, with $\beta$ taking values from 1 to $p-1$. Furthermore, assume that in each case the fusion results in $j=1$. The resulting state will be, up to normalization,

$$
\propto \sum_{i=1}^{p-1}\left(\prod_{\beta=1}^{p-1} F_{\beta i \rightarrow 1}\right) \psi_{i}|i\rangle \propto \sum_{i=1}^{p-1} \psi_{i}|i\rangle,
$$

where we have used the fact that multiplication by $i$, modulo $p$, is just a rearrangement of the values of $\beta$. 
The above procedure is a probabilistic projection onto $\mathcal{K}$ $=|0\rangle^{\perp}$. As usual, if we do not obtain $j=1$ as the result of each measurement, we just discard the state being projected.

What is the probability of success of the above procedure? The probability for obtaining $j=1$ on the first try is

$$
P_{j=1}=\sum_{i=1}^{p-1}\left|F_{i \rightarrow 1} \psi_{i}\right|^{2} \geqslant \min _{i>0}\left(\left|F_{i \rightarrow 1}\right|^{2}\right) \sum_{i=1}^{p-1}\left|\psi_{i}\right|^{2} .
$$

On subsequent measurements, the state has previously been projected to $|0\rangle^{\perp}$ and renormalized. Therefore the probability of success for each trial is simply bounded by

$$
P_{j=1} \geqslant \min _{i>0}\left(\left|F_{i \rightarrow 1}\right|^{2}\right) .
$$

The total probability of success is just the product of these quantities. In particular, the probability $p_{P P}$ associated with the probabilistic projection can be bounded by

$$
p_{P P} \geqslant \min _{i>0}\left(\left|F_{i \rightarrow 1}\right|^{2}\right)^{p-1}>0,
$$

where we used the fact that $\left|F_{i \rightarrow j}\right|>0$ for $i>0$.

Of course, the above is a underestimation of the probability of obtaining a good projection. For example, if all the results $j$ were equal to some fixed $j>1$, the same argument would show that a correct projection was obtained. Furthermore, there are many other ways in which the relative phases and amplitudes can cancel out. A classical computer, with knowledge of the values of $F_{i \rightarrow j}$, can keep repeating the procedure until such a cancellation occurs. The computer would also be required to stop after a long sequence of $j$ $=0$ results, in which case the state would have been projected onto $|0\rangle$.

In the end, as long as $p_{P P}$ is fixed and finite, we have produced the desired probabilistic projection to the space $|0\rangle^{\perp}$. Different values of $p_{P P}$ will just affect the complexity of an algorithm as a multiplicative constant. Furthermore, for the small groups that are likely to appear in the laboratory, $p_{P P}$ should be reasonably large. For example, in the case of $G=S_{3}, p_{P P}$ can be made exponentially close to 1 in the number of measurements.

It should be noted that because we are working with qudits of dimension $d=p$ and the semidirect product requires $p>q \geqslant 2$, the above projection will always be a nontrivial operation. In fact, it will always be powerful enough to complete a universal gate set.

At this point, all that remains to be done is to prove the universality of the gates constructed from the basic anyon operations. This will be the subject of Sec. IV. However, before closing this section, we shall discuss some issues regarding the measurability of electric charges and look at some alternative operations that could have been employed.

\section{H. On the measurement of one-dimensional representations}

The feasibility and accuracy of the probabilistic projection onto $|0\rangle^{\perp}$ depend crucially on being able to identify electric charges carrying one-dimensional representations.
However, these charges have a special property that makes them hard to identify: when only using braiding, a onedimensional representation is indistinguishable from the vacuum.

The reason behind the above difficulty is that onedimensional representations of a group $G$ are constant on conjugacy classes of $G$. Therefore, a magnetic charge that is braided around one of these electric charges will have its state change by an overall phase. These global phases are not measurable in quantum mechanics.

Of course, an interference experiment would produce a measurement of the charge. The standard double-slit experiment, with the electric charge located in between the slits, will produce a pattern on the screen that depends on the representation of the electric charge. However, during the experiment, the anyon will be in a superposition of spatial positions which is no longer protected from decoherence by topology. Since the interference experiment can be repeated many times without affecting the electric charge, this may not necessarily be a problem. However, it does involve working in a regime where the anyons can be treated as waves rather than particles.

On the bright side, these electric charges can also be detected by fusion, assuming the availability of electric charge ancillas with one-dimensional representations. Their fusion rules are particularly simple because these states have a onedimensional internal Hilbert space. Furthermore, their fusions always produce unique results. If $\gamma(g)$ and $\gamma^{\prime}(g)$ are two one-dimensional representations of a group $G$, then the fusion of the electric charges carrying these representations produces a charge of representation $\gamma^{\prime \prime}(g)=\gamma(g) \gamma^{\prime}(g)$. A charge will only fuse into the vacuum when fused with its conjugate representation. Therefore, after a series of fusions that end up producing the vacuum state, we can determine the representation of the original electric charge.

In fact, for groups with $q=2$ such as $S_{3}$, there is a further simplification. In these groups there are only two onedimensional representations: the vacuum and sign representations. Since the fusion of $\left|R\left(a^{i}\right)\right\rangle_{R}$ produces a onedimensional charge, if it does not fuse into the vacuum, then it must have produced the sign charge. Therefore, for these groups, we do not even require one-dimensional electric charge ancillas.

\section{Other possibilities}

In this section, we will briefly discuss one last possibility for producing useful operations: using the products of fusion. Though not strictly needed to complete a universal gate set, this subsection is an interesting study of alternative operations and the effects of decoherence during fusion.

At first sight, it appears that the projection onto $|0\rangle^{\perp}$ can be done without using electric charges with the following procedure: first fuse one anyon from the state to be measured with a $b^{-1}$ flux. Only the $|0\rangle$ state can fuse into the vacuum. If an anyon remains, fuse again with a $b$ flux to restore it to its previous state and pair it with its old partner. Repeating the procedure multiple times (because the $|0\rangle$ could turn into 
an electric charge rather than the vacuum) yields the desired projection.

There are, however, two problems with the above construction. The first, and smaller, problem is that when fusing with $b^{-1}$ or with $b$ we could be turning our magnetic charges into dyons. For groups of the form $\mathbb{Z}_{p} \times_{\theta} \mathbb{Z}_{q}$ the dyonic electric charges are all one dimensional, however, and will therefore have no effect on braiding, as discussed in the previous section. The probabilities of fusion into the vacuum will be reduced and, therefore, so will the respective projection probabilities, but they will still remain nonzero. In fact, a careful examination of the operations constructed so far shows that they work with a probabilistic mixture of dyons and regular magnetic charges.

The second and larger problem, though, is decoherence. The fluxes $a^{i} b a^{-i} b^{-1}=a^{i(1-t)}$ belong, in general, to different conjugacy classes and therefore different superselection sectors. When the quantum state is encoded in this form, it is susceptible to decohere into the different superselection sectors.

When does this decoherence occur? It occurs during fusion. In general, fusion takes two $n$-dimensional Hilbert spaces $\mathcal{H}$ and maps them to one: $\mathcal{H}_{1} \times \mathcal{H}_{2} \rightarrow \mathcal{H}_{3}$. But quantum mechanics is unitary; therefore, what must really be happening is a mapping to a tensor product of $\mathcal{H}$ and the environment: $\mathcal{H}_{1} \times \mathcal{H}_{2} \rightarrow \mathcal{H}_{3} \times \mathcal{E}$. When two states are mapped onto new states that are orthogonal in the environment subspace, decoherence occurs.

How do we know if states will have orthogonal environment components after fusion? If two states belong to the same superselection sectors, they are related by symmetry, which protects them from decoherence. This may not be the case when they come from different superselection sectors, though.

For example, consider the states $\left|a^{i}\right\rangle \otimes\left|a^{j} b\right\rangle$ for $i$ and $j$ between 0 and $p-1$, where the kets will denote single anyons in this paragraph and the next. States of different $j$ are all in the same conjugacy class, but states of different $i$ are grouped into conjugacy classes of $q$ elements (except for $i$ $=0$, which is its own conjugacy class). In total, we are talking about $p^{2}$ states.

These states fuse into the states with flux $a^{k} b$ for $0 \leqslant k$ $<p$. The resulting states may also have one of $q$ electric charges. In total, we fuse into a space containing $p q$ states. Since $p q<p^{2}$, what must be happening is that different conjugacy classes are mapped to states that are orthogonal in the environment subspace.

Note that the decoherence seems to occur when fusing out of a state made up of different superselection sectors. However, fusion is the only operation that could have measured the relative phase between the sectors, and it clearly does not. Therefore, it is acceptable to assume that the decoherence occurs as soon as states are mapped into different superselection sectors.

Returning to the question of alternative implementations of the projection onto $|0\rangle^{\perp}$, it is clear that the procedure described above does not achieve its goals without causing decoherence in the general case. However, in the special case when $q=p-1$, the nontrivial powers of $a$ form one conju- gacy class. Therefore, the above trick can produce a projection onto $|0\rangle^{\perp}$ using only magnetic charges. Of course, $q$ $=p-1$ only holds for $G=S_{3}$.

For other groups, the operation could become useful if we could tell into which superselection sector the state decohered, producing a probabilistic projection onto a smaller space. The smaller projections may also be computationally powerful. However, since we have completed a universal gate set without the results of this subsection, we shall work on proving universality from the previously constructed gates, rather than pursuing this matter further.

\section{GATE-SET UNIVERSALITY}

The goal of this section is to prove the universality of the following qudit gate set, which includes measurements (i) controlled- $X$ gate, (ii) probabilistic projection onto $|0\rangle$, (iii) probabilistic projection onto $|\widetilde{0}\rangle$, and (iv) probabilistic projection onto $|0\rangle^{\perp}$, where we assume that the qudits are of dimension $d>2$, with $d$ prime. The first requirement on $d$ is needed to make the gate set universal, whereas the second one will allow us to relate this gate set to Gottesman's gate set [8]. The above gate set must be supplemented by a controlling computer capable of universal classical computation.

The above gates were selected as those arising naturally from the anyons based on the groups $\mathbb{Z}_{p} \times_{\theta} \mathbb{Z}_{q}$. The proof of universality of the above gate set is the last step needed to show that universal quantum computation is feasible with these anyons.

The proof of universality will proceed in two steps. In the first step we will turn the second and third gates into proper measurements in the $Z$ and $X$ bases. Most of the methods of the first step were described while building computation with nonsolvable anyons [1]. The second step involves using the probabilistic projection onto $|0\rangle^{\perp}$ to construct magic states that complete the universal gate set. This is the new element needed to achieve universality with solvable anyons.

\section{A. Nondestructive measurement of $Z$ and $X$}

By the end of this subsection we will have constructed measurements in the $Z$ and $X$ bases. These measurements will be nondestructive in the sense that if result $i$ was obtained, the measured qudit will be in state $|i\rangle$ or $|\tilde{i}\rangle$, respectively. Because the measurements in question are complete, the nondestructive requirement can be achieved by having ancillas for every eigenstate of $X$ and $Z$, and then using the controlled- $X$ gate to swap the ancillas into the computational space.

The construction begins by producing a set of basic ancillas. Along the way we will also produce the $X$ and $Z$ unitary gates.

\section{1. $|0\rangle$ and $|\widetilde{0}\rangle$ ancillas}

Clearly, given $|0\rangle$ ancillas we can use the third gate to produce $|\widetilde{0}\rangle$ ancillas. Similarly, given $|\widetilde{0}\rangle$ ancillas we can use the second gate to produce $|0\rangle$ ancillas. Therefore, if the initial state of the quantum computer overlaps with either state, we can produce both kinds of ancilla. 
Usually, the initial state of the quantum computer is $|0\rangle$. However, by using the controlled- $X$ gate, in combination with the projections onto $|0\rangle$, we can obtain these states no matter what the qudits are initialized to. The procedure is just to apply a controlled- $X^{-1}$ gate (equivalent to $d-1$ controlled- $X$ gates) to two qudits and then project the target to the $|0\rangle$ space. If the initial state had some overlap with any of the states $|i\rangle \otimes|i\rangle$, then this produces the desired ancillas. Furthermore, even if we allow states that are initially entangled, once we involve more than $d$ qudits, at least one pair must have an overlap with the diagonal states. Therefore, $|0\rangle$ states can always be produced.

Henceforth, we shall assume an ample supply of $|0\rangle$ and $|\widetilde{0}\rangle$ ancillas.

\section{2. $|1\rangle$ states, $|\widetilde{1}\rangle$ states; $X$ gates, $Z$ gates}

The next step is to produce $|1\rangle$ and $|\widetilde{1}\rangle$ ancillas. The importance of these ancillas is that they will break the symmetry currently present in the one-qudit Hilbert space.

There are two symmetries in the Hilbert space that are not fixed by the basic four gates of our set. The first symmetry is a relabeling $|i x\rangle \rightarrow|i\rangle$, calculated modulo $d$, for some $0<x$ $<d$. The second, is the relabeling $Z^{y} \rightarrow Z$, for integer $0<y$ $<d$. For fixed $x$, the second symmetry is a relabeling of our $d$ th root of unity $\omega$ by $\omega^{y} \rightarrow \omega$ and a relabeling $|\widetilde{j y}\rangle \rightarrow|\widetilde{j}\rangle$.

Therefore, given an ancilla in a state $|x\rangle$, with $x>0$, we can just rename it so that it becomes a $|1\rangle$ ancilla. Similarly, given an ancilla in a state $|\tilde{y}\rangle, y>0$, we can relabel it as $|\tilde{1}\rangle$. In fact, both can be done simultaneously in a consistent fashion, even if we do not know the values of $x$ and $y$.

The initial states $|x\rangle$ and $|\tilde{y}\rangle$ can be obtained from two maximally mixed states. The maximally mixed states can be described either as a state $|x\rangle$ with $x$ chosen at random or a state $|\tilde{y}\rangle$ with $y$ chosen at random. Therefore, two maximally mixed states serve our purpose as long as we do not obtain $x=0$ or $y=0$. These two bad cases will be detected below, in which case the process can be restarted with two new mixed states.

To produce the maximally mixed states we apply a controlled- $X$ gate with $|\widetilde{0}\rangle$ as source and $|0\rangle$ as target. The result is a maximally entangled state, which can be turned into a maximally mixed state by discarding one of the two qudits. Two of these mixed states will serve as our ancillas.

Given our two ancillas, which we have now labeled $|1\rangle$ and $|\widetilde{1}\rangle$, we can build $X$ and $Z$ gates which are consistent with the new labeling. The $X$ gate is clearly just a controlled$X$ gate with a $|1\rangle$ state as control, whereas the $Z$ gate is just a controlled- $X$ gate with a $|\widetilde{1}\rangle$ as target. The less familiar second construction is just a specific case of the following circuit:

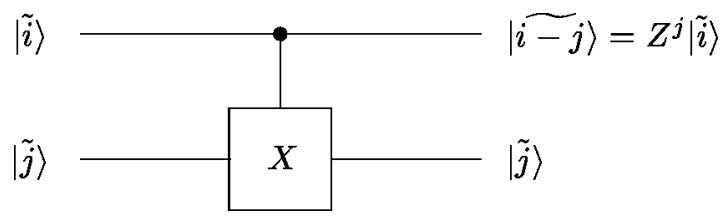

At this point, if we were unlucky enough to get $x=0$ or $y=0$, then one of the transformations $X$ or $Z$ will be the identity operator. This can easily be checked by applying them to $|0\rangle$ or $|\widetilde{0}\rangle$ ancillas and then using the available probabilistic projections.

The $X$ and $Z$ gates can also be used to produce a reservoir of $|1\rangle$ and $|\widetilde{1}\rangle$ ancillas that will be consistent with the original states. Two elements in the reservoir can also be compared, for example, by applying a $Z$ built from one ancilla followed by a $Z^{-1}$ built from the other. Therefore, even if the states were to decay over time, by using majority voting the damaged states can be weeded out.

In some cases, the one-qubit Hilbert spaces do have natural $|1\rangle$ or $|\widetilde{1}\rangle$ states, which implies a natural way of measuring or obtaining such states. For those systems, either the natural ancillas or the arbitrary ones constructed above can be used. For example, for the anyons $|1\rangle=\left|a b a^{-1}\right\rangle$. However, choosing a different $|1\rangle$ state is equivalent to choosing a different element $a$.

\section{Measurements of $Z$ and $X$}

At this point all the elements are in place to produce measurements in either the $Z$ basis or the $X$ basis.

The key element of the $X$ basis measurement is the circuit

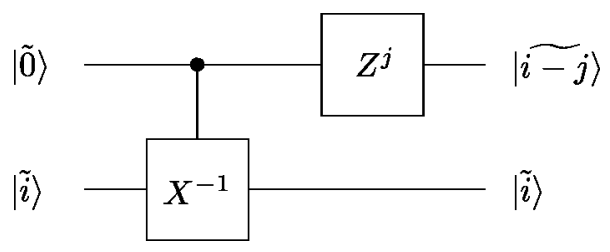

applied to a $|\widetilde{0}\rangle$ ancilla and the state to be measured. If the above circuit is repeated many times, each time with a different $|\widetilde{0}\rangle$ ancilla and with $j$ varying from 0 to $d-1$, we obtain the transformation

$$
\begin{aligned}
\sum_{i} \beta_{i}|\tilde{i}\rangle \rightarrow & \sum_{i} \beta_{i}|\tilde{i}\rangle \otimes|\tilde{i}\rangle \cdots \otimes|\widetilde{i-1}\rangle \otimes|\widetilde{i-1}\rangle \cdots \\
& \otimes|\widetilde{i-d+1}\rangle
\end{aligned}
$$

A probabilistic projection onto $|\widetilde{0}\rangle$ can then be applied to each qudit. If one of the qudits of the form $|\widetilde{i-j}\rangle$ projects onto the space $|\widetilde{0}\rangle$, then the outcome of the measurement is $j$.

Note that because of the one-sided error model of the probabilistic projection, an erroneous measurement result can never be obtained, no matter how small $p_{P P}$ is. The worst possible outcome is that after all the qudits have been measured, no conclusion can be reached. Of course, a standard small two-sided probability of error can also be made exponentially small by using enough qudits in the above measurement.

The measurement in the $Z$ basis proceeds similarly, where the transformation 


$$
\begin{gathered}
\sum_{i} \alpha_{i}|i\rangle \rightarrow \sum_{i} \alpha_{i}|i\rangle \otimes|i\rangle \cdots \otimes|i-1\rangle \otimes|i-1\rangle \cdots \\
\otimes|i-d+1\rangle
\end{gathered}
$$

is performed using the $X$ and controlled- $X$ gates, followed by a probabilistic projection onto $|0\rangle$.

Finally, the above measurements can be performed nondestructively, by projecting all but one of the qudits. Alternatively, the eigenstates of $X$ and $Z$ can be directly constructed from these gates and $|0\rangle$ or $|\widetilde{0}\rangle$ eigenstates.

\section{B. Completing the gate set}

So far, we have only shown that our gates can realize operations in the Clifford group. In order to achieve universal quantum computation we need to complete the gate set with an operation outside the Clifford group.

It was shown in Ref. [1] that the Toffoli gate, combined with measurements in the $X$ and $Z$ bases, is universal for quantum computation. Therefore, a successful construction of the Toffoli out of our gate set will prove it universal. The Toffoli gate will be constructed out of the previously described operations, together with the thus far unused probabilistic projection onto $|0\rangle^{\perp}$.

In addition to producing measurement gates, probabilistic projections are particularly useful for preparing magic states, which are ancillas whose use allows us to perform new gates such as the Toffoli. In particular, we shall show that we can produce the two magic states

$$
\begin{array}{r}
\left|\phi_{M 1}\right\rangle=\frac{1}{d} \sum_{i, j}|i\rangle \otimes|j\rangle \otimes|i j\rangle, \\
\left|\phi_{M 2}\right\rangle=\frac{1}{d} \sum_{i, j} \omega^{\delta_{i, 0} \delta_{j, 0}|i\rangle} \otimes|j\rangle,
\end{array}
$$

where $\delta_{i, j}$ is the Kronecker delta function. The first of these states produces the Toffoli gate up to some errors in the Clifford group. The second magic state allows us to correct these errors and, in fact, allows the construction of the complete Clifford group even without the use of the first magic state.

We shall begin by discussing how to use each of the magic states and then afterwards turn to the task of describing their construction out of the available operations.

\section{Using $\left|\phi_{M 1}\right\rangle$}

The magic state $\left|\phi_{M 1}\right\rangle$ and its use in producing the Toffoli gate was first introduced by Shor [9] and generalized to qudits in Ref. [8]. We shall give a brief description of its use in order to give an account of the exact Clifford group operations needed in the last step as corrections.

The procedure begins with a general state

$$
|\Psi\rangle=\sum_{a, b, c} \psi_{a, b, c}|a\rangle \otimes|b\rangle \otimes|c\rangle,
$$

to which an ancilla $\left|\phi_{M 1}\right\rangle$ is appended. A controlled- $X^{-1}$ gate is applied to the first data qudit with the first ancilla qudit as control. Similarly, a controlled- $X^{-1}$ gate is applied to the second data qudit from the second ancilla qudit, and a controlled- $X$ gate is applied to the third ancilla qudit, from the third data qudit. The first two data qudits are then measured in the $Z$ basis and the third data qudit is measured in the $X$ basis. If the results of the measurements are $\alpha, \beta$, and $\gamma$, respectively, then the remaining qudits are left in the state

$$
\sum_{a, b, c} \psi_{a, b, c} \omega^{\gamma c}|a-\alpha\rangle \otimes|b-\beta\rangle \otimes|(a-\alpha)(b-\beta)+c\rangle .
$$

The corrections begin by applying an $X^{\alpha} \otimes X^{\beta} \otimes X^{-\alpha \beta}$ gate followed by a controlled- $X^{\beta}$ gate from the first qudit to the third qudit and a controlled- $X^{\alpha}$ gate from the second qudit to the third qudit. The state then becomes

$$
\sum_{a, b, c} \psi_{a, b, c} \omega^{\gamma c}|a\rangle \otimes|b\rangle \otimes|a b+c\rangle .
$$

All that is needed to complete the Toffoli gate is a $Z^{-\gamma}$ gate applied to the third qudit and a phase $\omega^{\gamma a b}$ applied to the first two qudits. Unfortunately, we must first build the latter transformation out of the second magic state.

\section{Using $\left|\phi_{M 2}\right\rangle$}

Once again, the magic state is appended to a pair of qudits. Now controlled- $X$ gates are applied with the data qudits as source and the ancilla qudits as targets. Then the ancilla qudits are measured in the computational basis. The outcomes $\alpha$ and $\beta$ will be uniformly distributed, and at the end we will have produced the transformation

$$
\sum_{a, b} \psi_{a, b}|a\rangle \otimes|b\rangle \rightarrow \sum_{a, b} \psi_{a, b} \omega^{\delta_{a, \alpha} \delta_{b, \beta}}|a\rangle \otimes|b\rangle
$$

This procedure randomly and uniformly chooses a computational basis state and multiplies it by a phase of $\omega$. Repeated application of this transformation will eventually yield any of the $d^{d^{2}}$ states of the form

$$
\sum_{a, b} \psi_{a, b} \omega^{f(a, b)}|a\rangle \otimes|b\rangle,
$$

where $f$ is an arbitrary integer-valued function. This process is effectively a classical random walk on a $d^{2}$-dimensional periodic lattice with $d^{d^{2}}$ nodes, where each use of a magic state is equivalent to taking one step. Because the lattice is finite, after a polynomially large number of steps the probability of not having arrived at least once at any one of the above states becomes exponentially small.

The final correction needed to complete the Toffoli gate was the phase transformation to the state with $f(a, b)$ $=\gamma a b$ and can therefore be realized using many copies of the second magic state. All that remains to prove universality is to describe the production of the magic states. 


\section{Making the magic states}

The final piece of the puzzle is the production of the magic states using the probabilistic projection onto $|0\rangle^{\perp}$.

Probabilistic projections onto a subspace are particularly powerful for making magic states, because it can be assumed that they successfully project into the subspace every time. That is, if the probabilistic projection does not project onto the desired subspace, the state is tossed out and the procedure is restarted from the beginning. Therefore, the probabilistic projection onto $|0\rangle^{\perp}$ effectively takes a state and removes the $|0\rangle$ component of the state:

$$
\sum_{i=0}^{d-1} \alpha_{i}|i\rangle \rightarrow A \sum_{i=1}^{d-1} \alpha_{i}|i\rangle
$$

where $A$ is some normalization constant. In fact, by combining this projection with the $X$ gate, we can remove any of the components $|i\rangle$.

The main strategy for this section is to construct a series of ancilla states of increasing complexity, until finally the desired magic states are obtained. At this point, we have a supply of ancillas of the form $|i\rangle$ and $|\tilde{j}\rangle$ for any $i$ and $j$. From the $|\widetilde{0}\rangle$ state we can also make the ancilla $(|0\rangle$ $+|1\rangle) / \sqrt{2}$ by removing all $|i\rangle$ for $i>1$ with the probabilistic projection.

The next step is to produce entangled two-qudit ancillas. Given a supply of ancillas of the form $|\Psi\rangle=\Sigma_{i} \psi_{i}|i\rangle$ we shall produce ancillas of the form

$$
\left|\Psi^{\prime}\right\rangle=\psi_{0}|0\rangle \otimes|1\rangle+\sum_{i=1}^{d-1} \psi_{i}|i\rangle \otimes|0\rangle=\sum_{i=0}^{d-1} \psi_{i}|i\rangle \otimes\left|\delta_{i, 0}\right\rangle .
$$

The procedure begins with the state

$$
|\Psi\rangle \otimes\left(\frac{1}{\sqrt{2}}|0\rangle+\frac{1}{\sqrt{2}}|1\rangle\right)=\frac{1}{\sqrt{2}} \sum_{i=0}^{d-1} \sum_{j=0}^{1} \psi_{i}|i\rangle \otimes|j\rangle,
$$

which in general has $2 d$ nonzero coefficients. We need to remove $d$ of these coefficients to obtain the state $\left|\Psi^{\prime}\right\rangle$.

The procedure, done once for each $k$ from 1 to $d-1$, is the following: First, apply a controlled- $X^{k}$ gate with the left qudit as source and the right qudit as target. Then, the right qudit is projected onto $|0\rangle^{\perp}$, and finally the controlled- $X^{k}$ gate is undone. For each $k$, we remove the components $|0\rangle$ $\otimes|0\rangle$ and $|-1 / k\rangle \otimes|1\rangle$. The operation $-1 / k$ is modulo $d$ as usual and ranges over all integers between 1 and $d-1$ because $d$ is prime. Therefore, given a supply of $|\Psi\rangle$ ancillas, we can probabilistically convert some of them into a supply of $\left|\Psi^{\prime}\right\rangle$ ancillas.

Note that the above procedure works even if the coefficients $\psi_{i}$ represent the state of other qudits, as long as these are ancilla qudits that can be tossed out if the projection procedure fails. In the same spirit, given ancillas of the form

$$
|\Phi\rangle=\sum_{i=0}^{1} \sum_{j=0}^{1} \phi_{i, j}|i\rangle \otimes|j\rangle
$$

we can produce the three-qudit ancillas

$$
\left|\Phi^{\prime}\right\rangle=\sum_{i=0}^{1} \sum_{j=0}^{1} \phi_{i, j}|i\rangle \otimes|j\rangle \otimes\left|\delta_{i, 0} \delta_{j, 0}\right\rangle
$$

The procedure again involves appending $(|0\rangle+|1\rangle) / \sqrt{2}$ to the ancilla $|\Phi\rangle$, which now generically has eight nonzero coefficients, and removing four of them. This is done with a set of controlled- $X$ gates with the third qudit as target, followed by a probabilistic projection of the third qudit onto $|0\rangle^{\perp}$, followed by the inverse controlled- $X$ gates. If we use two controlled $-X^{-1}$ gates controlled by the first two qudits respectively, the projection will remove the components with labels $|0\rangle|0\rangle|0\rangle,|1\rangle|0\rangle|1\rangle$, and $|0\rangle|1\rangle|1\rangle$. In addition, using two controlled- $X^{(d-1) / 2}$ gates, we remove $|1\rangle|1\rangle|1\rangle$ and $|0\rangle|0\rangle|0\rangle$ (again). These are the four states that need to be removed to produce the ancilla $\left|\Phi^{\prime}\right\rangle$.

The above two procedures allow us to finally produce the desired magic states. Starting with $|\widetilde{0}\rangle \otimes|\widetilde{0}\rangle$, we apply the first procedure to each ancilla and then apply the second procedure to the appended qudits. The resulting state is

$$
\frac{1}{d} \sum_{i=0}^{d-1} \sum_{j=0}^{d-1}|i\rangle \otimes|j\rangle \otimes\left|\delta_{i, 0}\right\rangle \otimes\left|\delta_{j, 0}\right\rangle \otimes\left|\delta_{i, 0} \delta_{j, 0}\right\rangle
$$

If the last three qudits are measured in the $X$ basis and the results are 0,0 , and 1 , respectively, then we will have produced the magic state $\left|\phi_{M 2}\right\rangle$.

In fact, measuring in the $X$ basis and only accepting if the result is zero is a convenient way to unentangle the system with temporary qudits. Therefore, the previously described procedures can be combined into the probabilistic transformation

$$
\sum_{i=0}^{d-1} \sum_{j=0}^{d-1} \psi_{i, j}|i\rangle \otimes|j\rangle \rightarrow \sum_{i=0}^{d-1} \sum_{j=0}^{d-1} \psi_{i, j}|i\rangle \otimes|j\rangle \otimes\left|\delta_{i, n} \delta_{j, m}\right\rangle,
$$

where the first state is either transformed into the second state with some nonzero probability or else it is damaged. The above transformation has only been discussed so far for $n=m=0$, but a trivial use of $X$ gates before and after the transformation will allow any $n$ and $m$.

Starting with $|\widetilde{0}\rangle \otimes|\widetilde{0}\rangle \otimes|0\rangle$, repeated application of the above procedure can produce 


$$
\begin{aligned}
& \frac{1}{d} \sum_{i=0}^{d-1} \sum_{j=0}^{d-1}|i\rangle \otimes|j\rangle \otimes|0\rangle \\
& \quad \rightarrow \frac{1}{d} \sum_{i=0}^{d-1} \sum_{j=0}^{d-1}|i\rangle \otimes|j\rangle \otimes|0\rangle \underset{n=0}{\otimes} \bigotimes_{m=0}^{d-1}\left|\delta_{i, n} \delta_{j, m}\right\rangle \\
& \rightarrow \frac{1}{d} \sum_{i=0}^{d-1} \sum_{j=0}^{d-1}|i\rangle \otimes|j\rangle \otimes|i j\rangle \underset{n=0}{\substack{d-1 \\
\otimes}} \bigotimes_{m=0}^{d-1}\left|\delta_{i, n} \delta_{j, m}\right\rangle
\end{aligned}
$$

where the second step involves only controlled- $X$ gates from the extra qudits to the third qudit. Erasing the extra qudits with a measurement in the $X$ basis and retaining only when all results are zero produces the desired magic state $\left|\phi_{M 1}\right\rangle$.

The construction of the magic states out of the probabilistic projection onto $|0\rangle^{\perp}$ completes the description of the Toffoli gate. Though the procedures of this section are far from optimal in terms of resources, they are sufficient to demonstrate universality. In particular, this completes the proof that universal quantum computation is feasible with anyons from groups of the form $\mathbb{Z}_{p} \times{ }_{\theta} \mathbb{Z}_{q}$.

\section{COMPUTATIONAL POWER OF MAGNETIC CHARGES}

In this section, we will be interested in classifying the computational power that can be achieved by braiding anyonic magnetic charges of a finite group. The range of operations that can be achieved by braiding is closely related to the structure of the group to which the magnetic charges belong. In particular, the possibility of realizing the operations of controlled- $X$ and Toffoli gates (equivalently a doubly-controlled- $X$ gate) are, respectively, related to the group properties of nilpotency and solvability. These standard properties of group theory will also be defined below.

There are certain important assumptions that go into the discussion in this section. First, we assume that each qubit is carried by a pair of anyons. Furthermore, we choose a computational basis corresponding to the states of definite flux (e.g., $|0\rangle=|g\rangle$ for some $g \in G$ ). We remind the reader at this point that the state $|g\rangle$ corresponds to an anyon of magnetic charge $g$ paired with a compensating anyon of charge $g^{-1}$ whose only purpose is to allow the pair to move through the system without introducing undesired correlations. Finally, we will restrict the discussion to operations that can be achieved by braiding magnetic charges. The consequences of lifting these restrictions will be discussed near the end of this section.

Let the fluxes corresponding to the zero and one states be the elements $b, b^{\prime} \in G$, respectively. If we desire a coherent superposition between the zero and one states, they must be in the same conjugacy class, and therefore $b^{\prime}=a b a^{-1}$ for some nontrivial $a \in G$. This is summarized by

$$
|0\rangle=|b\rangle, \quad|1\rangle=\left|b^{\prime}\right\rangle=\left|a b a^{-1}\right\rangle .
$$

Even if the basis in use is a qudit basis, with additional states, we will only concern ourselves with states that have support on the above two basis vectors.
Consider now a pair of these states. We are interested in the operations that can be achieved by conjugating the second state by the flux of the first state, with the help of ancillas. Let $g \in\left\{b, b^{\prime}\right\}$ be the flux of the first state. The most general conjugation possible is by a function of the form

$$
\begin{aligned}
f(g) & =c_{1} g c_{2} g c_{3} g \cdots g c_{n} \\
& =\left(d_{1} g^{\prime} d_{1}^{-1}\right)\left(d_{2} g^{\prime} d_{2}^{-1}\right) \cdots\left(d_{n-1} g^{\prime} d_{n-1}^{-1}\right) d_{n}
\end{aligned}
$$

for some fixed elements $\left\{c_{i}\right\} \in G$. In the second line, the expression has been rewritten in terms of $g^{\prime}=g b^{-1}$ and new elements $\left\{d_{i}\right\} \in G$ which can easily be determined in terms of $\left\{c_{i}\right\} \in G$. For example, $d_{2}=c_{1} b c_{2}$.

The power of the second line is that it expresses the conjugation as a composition of two basic operations. The first is a conjugation by an ancilla with flux $d_{n}$ and is independent of the state of the first qubit. The second is conjugation by a product of conjugates of $g^{\prime}$, which was defined so that if $g$ $=b$, then $g^{\prime}=1$ and the product of its conjugates is trivial. In the other case, if $g=b^{\prime}$, then $g^{\prime}=[a, b] \equiv a b a^{-1} b^{-1}$ and the operation is conjugation by a product of conjugates of $[a, b]$.

We define $\mathcal{C}_{G}(x)$ as the conjugacy class of $x$ in $G$ and $\mathcal{C}_{G}^{\#}(x)$ as the group generated by the elements in $\mathcal{C}_{G}(x)$. The operations discussed so far are conjugation by fixed elements in $G$ and controlled conjugation by elements in $\mathcal{C}_{G}^{\#}([a, b])$.

The most natural controlled operation is the logical controlled- $X$ gate, which acts as a controlled conjugation by $a$. Naturally, if $a^{2} \neq 1$, then we could arrive at the qudit state $|2\rangle=\left|a^{2} b a^{-2}\right\rangle$. However, our interest lies in proving that certain groups cannot produce a controlled- $X$ gate, in which case it is sufficient to prove that a controlled conjugation by $a$ is unfeasible.

It seems that a requirement for a controlled conjugation by $a$ is the existence of elements $a, b$ such that $a$ $\in \mathcal{C}_{G}^{\#}([a, b])$. There is a potential loophole in the argument, though, because different qubits could use different basis fluxes. The target qubit could use $b_{2}$ as the zero state and $a_{2} b_{2} a_{2}^{-1}$ as the one state. If $a_{2} \in \mathcal{C}_{G}^{\#}\left(\left[a_{1}, b_{1}\right]\right)$, then the controlled- $X$ gate would be possible. Considering many qubits requires a sequence of nontrivial elements $\left\{a_{i}\right\}$ and $\left\{b_{i}\right\}$ which satisfy, at a minimum, the conditions

$$
a_{i+1} \in \mathcal{C}_{G}^{\#}\left(\left[a_{i}, b_{i}\right]\right)
$$

The above equations are related to the series of subgroups of $G$, defined by

$$
G^{((j+1))}=\left[G^{((j))}, G\right],
$$

with base case $G^{((0))}=G$. By definition, if $a_{i} \in G^{((j))}$, then $\left[a_{i}, b_{i}\right] \in G^{((j+1))}$. Furthermore, since the group $G^{((j+1))}$ is normal in $G$, the requirement on $a_{i+1}$ reads

$$
a_{i+1} \in \mathcal{C}_{G}^{\#}\left(\left[a_{i}, b_{i}\right]\right) \subset G^{((j+1))} .
$$

Of course, $a_{1} \in G^{((0))}=G$. Therefore, repeating the above argument shows that a controlled- $X$ gate requires $a_{i}$ $\in G^{((i-1))}$ with $a_{i} \neq 1$ for every $i \geqslant 1$. 
Given that $G$ is finite and $G^{((j+1))} \subset G^{((j))}$, the series must converge after a finite number of subgroups to some final subgroup $G^{((\infty))}$. The final subgroup can either be trivial or nontrivial. The groups with $G^{((\infty))}=\{1\}$ are called nilpotent. The conclusion thus far is that nilpotent groups cannot implement a controlled- $X$ gate by braiding. The inverse of this statement-i.e., that groups that are not nilpotent can implement the controlled- $X$ gate-will be shown in Sec. VI.

\section{A. Conjugations with multiple sources}

A similar analysis can be used to study the relationship between group structure and gates produced using multiple qubits as sources of conjugation. Clearly any group that is not nilpotent can produce a series of controlled- $X$ gates with different sources. However, certain groups are capable of producing much more powerful gates such as the Toffoli, which is universal for classical computation.

In the rest of this section we shall prove that groups that are solvable cannot produce a Toffoli gate, or equivalently universal classical computation, by braiding magnetic charges. This connection between universality for classical computation and nonsolvability had been previously identified by Barrington [10] in 1989. Though we shall mostly be interested in groups that are solvable, this result will place limits on the power that we can expect to obtain from braiding magnetic charges.

Just as above, the most general conjugation with $m$ sources is the conjugation by a function of the form

$$
\begin{aligned}
f\left(g_{1}, \ldots, g_{m}\right)= & \left(d_{1} g_{i_{1}}^{\prime} d_{1}^{-1}\right) \\
& \times\left(d_{2} g_{i_{2}}^{\prime} d_{2}^{-1}\right) \cdots\left(d_{n-1} g_{i_{n-1}}^{\prime} d_{n-1}^{-1}\right) d_{n},
\end{aligned}
$$

where $g_{i}^{\prime}=g_{i} b^{-1}$ and the indices $i$ take values from 1 to $m$. For brevity, we assume that all qubits are expressed in the same basis, though the general case would not be very different.

The Toffoli gate is simply a conjugation by a function $f_{T}\left(g_{1}^{\prime}, g_{2}^{\prime}\right)$, such that

$$
f_{T}\left(c^{k}, c^{l}\right)=a^{k l}
$$

which has been expressed as a function of $g_{i}^{\prime}$ and where we introduced $c \equiv[a, b]$. In order to produce the Toffoli gate using conjugation alone, we must be able to express the above equation in the form of Eq. (80) with $m=2$. We shall show that this is not possible for a solvable group.

For $m=2$, Eq. (80) is a product of conjugates of $g_{1}^{\prime}$ and $g_{2}^{\prime}$. We can rewrite it by moving all the conjugates of $g_{1}^{\prime}$ to the left and all the conjugates of $g_{2}^{\prime}$ to the right. In the center we will pick up factors of the form $\left[d_{i} g_{1}^{\prime} d_{i}^{-1}, d_{j} g_{2}^{\prime} d_{j}^{-1}\right]$ and commutators of commutators, and so on. In the end we will obtain

$$
f\left(g_{1}^{\prime}, g_{2}^{\prime}\right)=f_{1}\left(g_{1}^{\prime}\right) f_{C}\left(g_{1}^{\prime}, g_{2}^{\prime}\right) f_{2}\left(g_{2}^{\prime}\right) d_{n}
$$

where $d_{n}$ is a constant element of $G, f_{i}$ is a product of conjugates of $g_{i}^{\prime}$, and $f_{C}\left(g_{1}^{\prime}, g_{2}^{\prime}\right)$ is the factor with all the commutators. The function $f_{C}$ has the property that $f_{C}\left(g_{1}^{\prime}, 1\right)$ $=f_{C}\left(1, g_{2}^{\prime}\right)=1$.

Setting $f=f_{T}$ implies the conditions

$$
\begin{gathered}
1=f(1,1)=d_{n}, \\
1=f(c, 1)=f_{1}(c) d_{n}, \\
1=f(1, c)=f_{2}(c) d_{n}, \\
a=f(c, c)=f_{1}(c) f_{C}(c, c) f_{2}(c) d_{n},
\end{gathered}
$$

which imply $f_{C}(c, c)=a$.

However, $f_{C}$ has the additional property that, if $N$ is a normal subgroup of $G$ containing $c$, then $f_{C}(c, c) \in[N, N]$. Furthermore, since $c=[a, b]$, the requirement on $c$ needed to express the Toffoli function in product form is

$$
c \in N \Rightarrow c \in[N, N]
$$

for any normal subgroup $N$. This condition is related to the series of subgroups defined by

$$
G^{(j+1)}=\left[G^{(j)}, G^{(j)}\right],
$$

again with base case $G^{(0)}=G$. Just as before, this series must converge to a final subgroup $G^{(\infty)}$. The groups where $G^{(\infty)}=\{1\}$ are known as solvable. Any group that is nilpotent is also solvable.

Because the subgroups $G^{(j)}$ are all normal in $G$, the requirement of Eq. (84) can only be satisfied if $c$, which by definition cannot be 1 , is contained in $G^{(\infty)}$. We have therefore shown that if the group is solvable, then the function $f_{T}$ cannot be expressed in product form, and therefore we cannot conjugate by it. This is true even if the target of conjugation is in a known state, which implies that even if we had used the target as a source of conjugations as well (i.e., by using it to conjugate ancillas and then using the ancillas) the Toffoli gate would still not be feasible by using only braiding of anyons from a solvable group.

The fact that the Toffoli gate can be produced for nonsolvable groups is a consequence of the results of Refs. [1] and [10] and will not be discussed here. In fact, the computational model discussed in this section resembles the nonuniform deterministic finite automata presented in Ref. [11]. For nonsolvable groups, the two models are almost identical. Nonetheless, for solvable groups, the magnetic charges presented in this section have significantly less computational power, because the zero and one states have to be represented by group elements in the same conjugacy class.

\section{B. Summary of computational power}

The results discussed so far have been summarized by Table I. For each type of group, it describes the computational operations that can be achieved through braiding of magnetic fluxes, as well as an example. The examples are the smallest group in the class, with the exception of the Abelian case where the trivial group could also be listed. For the 
TABLE I. Computational power achieved by conjugation for different groups.

\begin{tabular}{ccccc}
\hline \hline Abelian & Nilpotent & Solvable & Example & Computational power \\
\hline yes & yes & yes & $Z_{2}$ & $I$ \\
no & yes & yes & $Q$ & $X$ \\
no & no & yes & $S_{3}$ & Controlled- $X$ \\
no & no & no & $A_{5}$ & Toffoli \\
\hline \hline
\end{tabular}

non-Abelian, nilpotent case there are two examples with eight elements: the dihedral group $D_{4}$ and the quaternionic group $Q$, which is listed in the table and has elements \pm 1 , $\pm i, \pm j, \pm k$.

The most basic case is when $G$ is Abelian, in which case it is also nilpotent and solvable. Clearly conjugation can only produce the identity transformation. In fact, every superselection sector consists of a one-dimensional Hilbert space, and therefore quantum information cannot even be stored in Abelian anyons in a topologically protected manner.

At the other extreme are anyons from nonsolvable groups. Universal classical computation can be accomplished through braiding, and universal quantum computation can be obtained by completing the gate set with measurements in the $X$ and $Z$ bases. The complete construction for this case is described in Ref. [1].

Anyons from groups that are solvable, but not nilpotent, can also be used for universal quantum computation, but the construction is more complicated. A controlled- $X$ gate can be constructed from flux braiding, and measurements in the $X$ and $Z$ bases can be constructed in a manner similar to the nonsolvable groups. However, to complete a universal gate set, fusions of electric charges must be employed. The proof of universality, along with the details of the gates, will be the subject of the rest of this paper.

Finally, anyons from groups that are nilpotent seem insufficient for universal computation. In the constructions for the non-nilpotent groups, the only operation that can produce entanglement between multiple qudits is the controlled- $X$ or Toffoli gates obtained by braiding fluxes. However, for nilpotent groups, braiding fluxes does not seem to yield an operation capable of producing entanglement. Either a new type of operation or a different basis must be used. Simple modifications to the basis, such as encoding a qudit on multiple anyons, are of no help. However, there are countless strange bases that are hard to discredit. For example, a lattice of electric charges could serve as a Hilbert space, with magnetic charges used to create or measure entanglement among the charges. Therefore, while the prospects of universal computation with nilpotent anyons seem bleak, the question remains open.

\section{SOLVABLE NON-NILPOTENT GROUPS}

In this section, we will prove that anyons based on a finite group that is solvable but not nilpotent are sufficient for universal quantum computation. The first step will be to decompose an arbitrary group $G$ that is solvable, but non-nilpotent, into a form similar to the previously studied $\mathbb{Z}_{p} \times_{\theta} \mathbb{Z}_{q}$ groups.
The proof of universality will then be a small generalization of the ideas presented in Sec. III.

\section{A. Group decomposition}

Let $G$ be a group as above and define $H \equiv G^{((\infty))}$ in terms of the series discussed in Sec. V. Because $G$ is non-nilpotent, $H$ is nontrivial, and because $G$ is solvable, $H \neq G$. Furthermore, $H$ is normal in $G$ and $G / H$ is nilpotent. The second fact is due to

$$
\begin{aligned}
(G / H)^{((i+1))} & =\left[(G / H)^{((i))},(G / H)\right]=\left[G^{((i))}, G\right] / H \\
& =G^{((i+1))} / H
\end{aligned}
$$

and therefore $(G / H)^{((\infty))}=H / H=\{1\}$.

Any nilpotent group can be written as the direct product of its Sylow $p$-groups, which are groups whose order is a prime power. Therefore,

$$
G / H=K_{q_{1}} \times K_{q_{2}} \times \cdots \times K_{q_{l}}
$$

where $K_{q}$ denotes a group of order $q^{m}$ for some prime $q$ and integer $m$. We further define $N K_{q_{i}}$ to be the lifting of $K_{q_{i}}$ to the full group $G$ that is $N K_{q_{i}} / N=K_{q_{i}}$. Note that to maintain consistency with the notation in Sec. III, the primes involved in these $p$-groups are labeled by the letter $q$.

Having fully characterized $G / H$, we turn to the study of $H$ itself. Let $N$ be the largest normal subgroup of $G$ that also satisfies $N \subset H$ and $N \neq H$. If more than one subgroup satisfies the above requirements, then let $N$ be any such subgroup. Because $H$ is finite, there must be at least one maximal subgroup.

We shall prove that $H / N=\mathbb{Z}_{p}^{n}$ for some prime $p$ and integer $n$. The basic idea is that working modulo $N, H / N$ is a normal subgroup of $G / N$. Furthermore, $H / N$ has no proper subgroups that are normal in $G / N$. In particular, this implies that $H / N$ is Abelian, because its commutator subgroup is a normal subgroup of $G / N$. Note that the possibility that the commutator subgroup of $H / N$ is equal to $H / N$ is excluded because $H / N$ is solvable.

For any $x \in H / N$ consider $\mathcal{C}_{G / N}^{\#}(x)$, the group generated by the conjugates of $x$ in $G / N$. This is a subgroup of $H / N$ and is normal in $G / N$. Therefore,

$$
\mathcal{C}_{G / N}^{\#}(x)=H / N, \quad \forall x \in H / N,
$$

which implies that all elements in $H / N$, with the exception of the identity, have the same order. That is because conjugates of $x$ have the same order as $x$ and a product of elements of order $k$ in an Abelian group must have order less than or equal to $k$. This concludes the proof that $H / N=Z_{p}^{n}$.

Thus far, we have the following tower of groups

$$
N \subset H \subset H K_{q_{i}} \subset G,
$$

where $N, H$, and $H K_{q_{i}}$ are all normal in $G$ and the group $H K_{q_{i}}$ can be any of the groups found above.

Because $(G / N)^{((\infty))}=H / N=Z_{p}^{n}$, the group $G / N$ is also solvable and non-nilpotent. However, its structure is simpler 
than that of the full group $G$. We shall therefore be interested in working modulo $N$ and shall denote groups modulo $N$ by a tilde. That is,

$$
\widetilde{G}=G / N, \quad \widetilde{H} K_{q_{i}}=H K_{q_{i}} / N, \quad \widetilde{H}=H / N=\mathbb{Z}_{p}^{n} .
$$

The final step is to study the relationship between $\widetilde{H}$ and the groups $\widetilde{H} K_{q_{i}}$. By construction, we know $[\widetilde{G}, \widetilde{H}]=\widetilde{H}$ $=Z_{p}^{n}$, but what about $\left[\widetilde{H} K_{q_{i}}, \widetilde{H}\right]$ ? Because both $\widetilde{H} K_{q_{i}}$ (for any $i$ ) and $\widetilde{H}$ are normal in $\widetilde{G},\left[\widetilde{H} K_{q_{i}}, \widetilde{H}\right]$ is normal in $\widetilde{G}$ and, furthermore, is contained in $\widetilde{H}$. But $N$ was defined to be the largest proper subgroup of $H$ that was normal in $\widetilde{G}$. Therefore $\widetilde{H}$ has no proper subgroups that are normal in $\widetilde{G}$, and $\left[\widetilde{H} K_{q_{i}}, \widetilde{H}\right]$ must be either the trivial group or all of $\widetilde{H}$.

If $q_{i}=p$, then $\widetilde{H} K_{q_{i}}$ is a $p$-group and, therefore, nilpotent. This means that $\left[\widetilde{H} K_{p}, \widetilde{H}\right] \neq \widetilde{H}$ and by the previous paragraph $\left[\widetilde{H} K_{p}, \widetilde{H}\right]=\{1\}$. The rest of the groups $\widetilde{H} K_{q_{i}}$ can either commute or not with $\widetilde{H}$. However, because $[\widetilde{G}, \widetilde{H}]$ $=\widetilde{H}$, at least one of them must not commute. Fix an $i$ such that $\left[\widetilde{H} K_{q_{i}}, \widetilde{H}\right]=\widetilde{H}$, and define $K=K_{q_{i}}, \widetilde{H} K=\widetilde{H} K_{q_{i}}$, and $q$ $=q_{i}$. This will be the group to take the place of $\mathbb{Z}_{q}$.

We would like to show that there exists an element $b$ $\in \widetilde{H} K$, such that $[b, \widetilde{H}]=\widetilde{H}$. Let $X$ be the stabilizer of $\widetilde{H}$ in $\widetilde{H} K$-that is, the largest subgroup of $\widetilde{H} K$ such that $[X, \widetilde{H}]$ $=1$. Clearly $H \subset X$ and $X \neq \widetilde{H} K$. Because $\widetilde{H} K / X$ is nilpotent, it has a nontrivial center. Let $b \in \widetilde{H} K$ be any element that projects, modulo $X$ to one of the nontrivial elements in the center. We will show that $[b, \widetilde{H}]$ is normal in $\widetilde{G}$, which implies $[b, \widetilde{H}]=\widetilde{H}$. The proof is that modulo $X$ (which is normal in $\widetilde{G}$ ), every element $g \in \widetilde{G}$ commutes with $b$. Therefore $g b g^{-1}=b x$ for some $x \in X$ and

$$
g[b, h] g^{-1}=b x h^{\prime} x^{-1} b^{-1} h^{\prime}-\underline{L} b h^{\prime} b^{-1} h^{\prime-1} \in[b, \widetilde{H}]
$$

for any $h \in \widetilde{H}$, where $h^{\prime}=g h g^{-1} \in \widetilde{H}$.

To summarize, working modulo $N$, we have the following tower of subgroups:

$$
\widetilde{H} \subset \widetilde{H} K \subset \widetilde{G},
$$

with $\widetilde{H}=Z_{p}^{n}$ for some prime $p$. Furthermore, $\widetilde{H} K / \widetilde{H}=K$ is a subgroup of order a power of $q$, for some prime $q$ not equal to $p$. Finally, $\exists b \in \widetilde{H} K$ such that $[b, \widetilde{H}]=\widetilde{H}$.

Note that this notation is consistent with the one used in Sec. III. That is, if $G=\mathbb{Z}_{p} \times{ }_{\theta} \mathbb{Z}_{q}$, then $N=\{1\}, H=\mathbb{Z}_{p}, K$ $=Z_{q}$, and the definitions of $p, q$, and $b$ would be consistent.

\section{B. Examples}

There are a few good examples to keep in mind that illustrate the potential new complications arising from groups with more structure than $\mathbb{Z}_{p} \times_{\theta} \mathbb{Z}_{q}$.
The first example is $A_{4}=Z_{2}^{2} \times_{\theta} Z_{3}$. The group can be described as $a_{1}=(12)(34), a_{2}=(23)(41), b=(123)$ with

$$
\begin{gathered}
a_{1}^{2}=a_{2}^{2}=1, \quad a_{1} a_{2}=a_{2} a_{1}, \\
b^{3}=1, \quad b a_{1}^{i} a_{2}^{j} b^{-1}=a_{1}^{j} a_{2}^{i+j} .
\end{gathered}
$$

For this group $N=\{1\}, H=Z_{2}^{2}$, and $K=Z_{3}$. Its most important feature is that $p=2$, which was not previously possible. Because $p=2$ implies working with qubits, these groups will be have to be handled specially.

The next example is $G=\left(\mathbb{Z}_{3}^{2}\right) \times{ }_{\theta}\left(\mathbb{Z}_{3} \times \mathbb{Z}_{2}\right)$. Let $a_{1}, a_{2}$ be the generators of $Z_{3}^{2}$, and let $b$ be the generator of $Z_{2}$ and $x$ be the generator of the remaining $\mathbb{Z}_{3}$. The semidirect product is defined by the conjugations

$$
b a_{1}^{i} a_{2}^{j} b^{-1}=a_{1}^{-i} a_{2}^{-j}, \quad x a_{1}^{i} a_{2}^{j} x^{-1}=a_{1}^{-j} a_{2}^{i-j} .
$$

For this group $H=Z_{3}^{2}$ because $[G, G]=[G, H]=H$. The subgroup generated by $a_{1} a_{2}^{-1}$ is normal in $G$ and therefore $N$ $=Z_{3}$. Finally $H / N=Z_{3}$ and $K=Z_{2}$. Note that $x$ commutes with $H$ modulo $N$, as discussed in the last section.

The final pair of examples illustrate the case where $K$ is non-Abelian. The examples are $\mathbb{Z}_{3}^{2} \times_{\theta} Q$ and $Z_{3}^{2} \times_{\theta} D_{4}$. Labeling the generators of $\mathbb{Z}_{3}^{2}$ by $a_{1}$ and $a_{2}$, the semidirect product for $\mathbb{Z}_{3}^{2} \times_{\theta} Q$ is defined by

$$
i a_{1}^{x} a_{2}^{y} i^{-1}=a_{1}^{y} a_{2}^{-x}, \quad j a_{1}^{x} a_{2}^{y} j^{-1}=a_{1}^{x+y} a_{2}^{x-y},
$$

where $\pm 1, \pm i, \pm j, \pm k$ are the standard quaternionic elements. For $Z_{3}^{2} \times_{\theta} D_{4}$ the semidirect product is defined by

$$
\beta a_{1}^{x} a_{2}^{y} \beta^{-1}=a_{1}^{y} a_{2}^{-x}, \quad \gamma a_{1}^{x} a_{2}^{y} \gamma^{-1}=a_{1}^{y} a_{2}^{x},
$$

where the relations $\beta^{4}=\gamma^{2}=1$ and $\gamma \beta \gamma=\beta^{-1}$ define $D_{4}$.

In both of the above cases $p=3, q=2, N=\{1\}$, and $H$ $=Z_{3}^{2}$. However, for $\mathbb{Z}_{3}^{2} \times_{\theta} Q$ the nontrivial elements of $H$ are conjugate to one another, and none of the nontrivial elements of $Q$ commute with any of the nontrivial elements of $H$. The $Z_{3}^{2} \times_{\theta} D_{4}$ case divides $H$ into three conjugacy classes (including the identity). Furthermore, each of the elements of the form $\beta^{i} \gamma$ commute with two nontrivial elements of $H$. These differences will become important when discussing the operations involving electric charges.

\section{C. $N$-invariant ancillas}

The first lesson from the above analysis is that we should work modulo $N$. That is, we want flux states labeled by elements of $\widetilde{G}=G / N$ that are invariant under $N$. The idea of $N$-invariant states was already discussed in Ref. [1] when generalizing simple non-Abelian anyons to nonsolvable ones, and therefore the discussion below will be brief.

A basis for the $N$-invariant magnetic fluxes is just $|g\rangle$ for $g \in \widetilde{G}$. The braiding and fusion properties of these states behave almost exactly as if the full group were $\widetilde{G}$ and these states were flux eigenstates. The only difference is that when fusing two anyons from pairs with opposite fluxes, the probability of disappearing into the vacuum is lower. 
Even producing anyons from the vacuum behaves correctly with respect to $N$ invariance. Pairs produced from the vacuum are naturally invariant under the full group $G$. Normally, when braiding with other states, this invariance will be broken. However, if the vacuum pair only interacts with $N$-invariant states, then the invariance under the group $N$ will remain.

At this point we will change our requirements for the physical system. Instead of requiring a reservoir of flux ancillas for every element of $G$, we will require a reservoir of $N$-invariant flux ancillas for every element of $\widetilde{G}$. This is likely a reasonable modification, as it appears that the latter ancillas are no harder to produce than the original ones.

It should be noted that, when working modulo $N$, the electric charges need no modification. That is, because $N$ is normal in $G$, any representation of $\widetilde{G}$ extends to a representation of $G$ that is invariant under $N$. Furthermore, fusing two $\mathrm{N}$-invariant electric charges must produce a new $\mathrm{N}$-invariant electric charge. Therefore, working with $N$-invariant electric charges simply involves working with a subset of the charges of the group $G$.

Given the above caveats, we can effectively replace the group $G$ with the group $\widetilde{G}=G / N$, which will be done without further comment for the rest of this section.

\section{Computational basis}

We will begin by defining an extended computational basis and discuss the operations that can be performed on this extended subspace. Toward the end of this section, a subset of these states will be singled out as the true computational basis.

Let $a_{1}, \ldots, a_{n}$ be a set of generators for $\widetilde{H}=Z_{p}^{n}$, and recall the definition of the element $b \in \widetilde{G}$. The extended computational basis consists of the states

$$
\left|i_{1}, \ldots, i_{n}\right\rangle \equiv\left|a_{1}^{i_{1}} \cdots a_{n}^{i_{n}} b a_{n}^{-i_{n}} \cdots a_{1}^{-i_{1}}\right\rangle,
$$

where each of the $i$ 's takes values from 0 to $p-1$.

To prove that the states are all distinct consider the map from $H \rightarrow H$ defined by

$$
[g, \cdot]: h \rightarrow[g, h]
$$

Because $\widetilde{H}$ is Abelian, this map is an homomorphism for any $g \in \widetilde{G}$. In particular, since $[b, \widetilde{H}]=\widetilde{H}$, the homomorphism defined by $[b, \cdot]$ is surjective and has trivial kernel. That is, no element of $\widetilde{H}$ commutes with $b$. But

$$
h b h^{-1}=h^{\prime} b h^{\prime}-\underline{\Rightarrow}\left(h^{\prime}-1 h\right) b\left(h^{\prime}-1 h\right)^{-1}=b
$$

for any elements $h, h^{\prime} \in \widetilde{H}$, which can only be true if $h$ $=h^{\prime}$.

\section{E. Basic operations}

The generalized controlled- $X$ gate is the transformation

$$
\begin{aligned}
\left|i_{1}, \ldots, i_{n}\right\rangle \otimes\left|j_{1}, \ldots, j_{n}\right\rangle \rightarrow & \left|i_{1}, \ldots, i_{n}\right\rangle \\
& \otimes\left|i_{1}+j_{1}, \ldots, i_{n}+j_{n}\right\rangle .
\end{aligned}
$$

It can be implemented as a conjugation of the second anyon by a function of the flux of the first anyon such that

$$
f\left(h b h^{-1}\right)=h
$$

for any $h \in \widetilde{H}$. Because the map $[b, \cdot]$ defined above is just a permutation of the elements of $\widetilde{H}$, it has a finite period (say, $l$ ). The desired function is

$$
f(g)=\left[\left[\left[g b^{-1}, b\right], b\right], \ldots, b\right],
$$

which consists of $l-1$ nested commutators. The final commutator needed to complete the period is the one formed in the expression $g b^{-1}$ when $g$ has the form $h b h^{-1}$.

At this point, one may wonder how does working modulo a normal subgroup $N$ affect the discussion regarding the computability of the controlled- $X$ gate. The controlled- $X$ gate can only be implemented because $\widetilde{G}$ is non-nilpotent. In a sense, $\widetilde{G}$ was constructed to be as small as possible, but still maintain the property of being non-nilpotent. On the other hand, if a group $G$ is nilpotent to begin with, then any subgroup or quotient group will also be nilpotent, and no controlled- $X$ gate can be constructed using braiding.

Using the same techniques as in Sec. III D, anyon fusions can be used to perform measurements. Fusion with $\left|b^{-1}\right\rangle$ ancillas produces a probabilistic projection onto $|0, \ldots, 0\rangle$. Fusing the two anyons that form a qudit is a probabilistic projection onto

$$
|\widetilde{0}, \ldots, \widetilde{0}\rangle \equiv \frac{1}{\sqrt{p^{n}}} \sum_{i_{1}=0}^{p-1} \ldots \sum_{i_{n}=0}^{p-1}\left|i_{1}, \ldots, i_{n}\right\rangle .
$$

As usual, to complete the probabilistic projection, these fusions must be supplemented by a reservoir of $|0, \ldots, 0\rangle$ and $|\widetilde{0}, \ldots, \widetilde{0}\rangle$ ancillas. The first case is trivial, because the existence of these ancillas has been assumed as one of the physical requirements of the system. The production of $|\widetilde{0}, \ldots, \widetilde{0}\rangle$ ancillas is more complicated and will occupy the rest of the subsection.

The procedure to distill $|\widetilde{0}, \ldots, \widetilde{0}\rangle$ states begins with a pair created from the vacuum and a $|0, \ldots, 0\rangle$ ancilla:

$$
|\mathrm{Vac}\rangle \otimes|0, \ldots, 0\rangle
$$

Using only braiding, an incomplete swap is applied to the state:

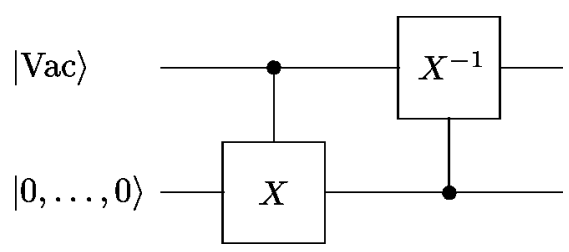


Once again, the circuit denotes the action of the conjugations on the computational basis, but their extension to the full Hilbert space needs to be discussed. After applying the necessary braidings to perform the circuit, the top state is fused with a $\left|b^{-1}\right\rangle$ ancilla. If the fusion does not produce the vacuum state, the final product is discarded and the procedure restarted from the beginning. Since conjugations cannot change the superselection sector, the only case that needs to be considered is when the vacuum state is created in the superselection sector that contains the computational subspace (i.e., the conjugacy class of $b$ ). In this superselection sector the vacuum state has the form

$$
|\operatorname{Vac}\rangle \propto|\widetilde{0}, \ldots, \widetilde{0}\rangle+\left|\Psi^{\perp}\right\rangle,
$$

where $\left|\Psi^{\perp}\right\rangle$ is a state in the space spanned by vectors of the form $\left|g g^{-1}\right\rangle$ that are not contained in the computational basis.

Because we want to guarantee that after the controlled- $X$ gate the state $|0, \ldots, 0\rangle$ remains in the computational subspace, we need the conjugation function to satisfy

$$
\begin{gathered}
f(\widetilde{G}) \in \widetilde{H} \\
f\left(h b h^{-1}\right)=h \quad \forall h \in \widetilde{H} .
\end{gathered}
$$

The second requirement can be satisfied by choosing $f$ as a sequence of commutators as in Eq. (102), as long as the number of commutators is one minus a multiple of $l$ (the period of $[b, \cdot])$. Furthermore, the result after $i$ commutators must be contained in $\widetilde{G}^{((i))}$. Because the series is finite, $\widetilde{G}^{((j))}=\widetilde{G}^{((\infty))}=\widetilde{H}$ for some finite $j$, and the first requirement can also be satisfied by defining $f$ to be a long enough sequence of commutators. Both requirements can be satisfied simultaneously by correctly choosing the number of commutators in the expression, and this completes the definition of the first controlled- $X$.

The second controlled- $X$ gate can be a regular controlled$X$ gate because in this case the control is known to be in the computational subspace. In the end, the vacuum state will be conjugated by an element of $\widetilde{H}$ and, therefore, can only have flux $b$ if it was originally in the computational subspace.

Having completed the construction of the $|\widetilde{0}, \ldots, \widetilde{0}\rangle$ ancillas, all that is required to complete a universal set of gates is an analog of the probabilistic projection onto $|0\rangle^{\perp}$ constructed out of fusions of electric charges.

\section{F. Using electric charges}

The ideal goal for this section would be the construction of the probabilistic projection onto $|0, \ldots, 0\rangle^{\perp}$ gate. Unfortunately, this is not possible for most groups. However, we will produce a pair of gates that have an equivalent computational power.

The first gate involves a nontrivial subgroup $\widetilde{\Lambda} \subset \widetilde{H}$, to be defined later, which could equal all of $\widetilde{H}$. Note that this subgroup defines a subspace of the computational space spanned by

$$
\left|\lambda b \lambda^{-1}\right\rangle
$$

for all elements $\lambda \in \tilde{\Lambda}$, which will also be denoted by $\tilde{\Lambda}$. The probabilistic projection onto $\tilde{\Lambda}$ will be the first gate.

The second gate is the probabilistic projection onto $|0, \ldots, 0\rangle^{\perp} \cap \tilde{\Lambda}$. This second gate can be though of as an application of the first gate, followed by a probabilistic projection onto $|0\rangle^{\perp}$ that only works on states contained in $\widetilde{\Lambda}$. For the moment, we will assume that the first gate can be implemented and work on the construction of the second gate.

The basic building block for this section involves working with the state to be measured $|\Psi\rangle$ and an electric charge pair in the vacuum state $|R(I)\rangle_{R}$ of some non-Abelian representation $R$. The state to be measured is contained in the computational basis and can therefore be expanded as

$$
|\Psi\rangle=\sum_{h \in \widetilde{H}} \psi_{h}\left|h b h^{-1}\right\rangle
$$

where, as in Sec. III G, the coefficients $\left\{\psi_{h}\right\}$ could be numbers or could denote the state of the rest of the system.

Using braiding, the state $|\Psi\rangle$ can be entangled with the electric charges. In particular, if $\phi(g)$ is a function constructed as a product of $g$ and fixed elements of $\widetilde{G}$, then the following transformation can be realized:

$$
|\Psi\rangle \otimes|R(I)\rangle_{R} \rightarrow \sum_{h \in \widetilde{H}} \psi_{h}\left|h b h^{-1}\right\rangle \otimes|R(\phi(h))\rangle_{R}
$$

Note that the state of the electric charge can depend on $\phi(h)$ rather than $\phi\left(h b h^{-1}\right)$ by composing with the function defined in Eq. (102). That is $\phi\left(f\left(h b h^{-1}\right)\right)=\phi(h)$.

Now the electric charge pair is fused together, and the resulting particle is measured. More specifically, in accordance with the discussion in Sec. III H, we just check whether the resulting particle belongs to some onedimensional representation labeled $\gamma$. If the charge $\gamma$ is detected, then the electric charge will have disentangled with the state being measured, because its internal Hilbert space is one dimensional. Furthermore, because each onedimensional representation occurs only once in the decomposition of $R \otimes R^{*}$, the state will be unentangled with the environment as well. The proof of the latter property uses Schur's lemma and the fact that if $\left|M_{1}\right\rangle_{R}$ and $\left|M_{2}\right\rangle_{R}$ always fuse into representation $\gamma$, then $\left|M_{1} M_{2}^{\dagger}\right\rangle_{R}$ will always fuse into the vacuum.

The result of the complete operation, when the outcome $\gamma$ is obtained, is the transformation

$$
|\Psi\rangle \rightarrow \sum_{h \in \widetilde{H}} F_{\phi(h) \rightarrow \gamma} \psi_{h}\left|h b h^{-1}\right\rangle
$$

where the state after the measurement has been left unnormalized. The coefficients $F_{h \rightarrow \gamma}$ depend implicitly on the original representation $R$ and will be defined carefully below. 
The above procedure can be repeated many times for different functions $\phi(g)$. If on each occurrence the outcome $\gamma$ is obtained, the resulting (unnormalized) state will be

$$
\sum_{h \in \widetilde{H}}\left(\prod_{\phi \in \Phi} F_{\phi(h) \rightarrow \gamma}\right) \psi_{h}\left|h b h^{-1}\right\rangle
$$

where $\Phi$ is the set of functions used. As usual, if the outcome $\gamma$ is not obtained on each instance, the state is discarded, and the probabilistic projection reports a projection onto the complement.

We assume that all functions in the set $\Phi$ are products of conjugates of the input, and therefore $\phi(I)=I$ for any $\phi$ $\in \Phi$. Because $|R(I)\rangle_{R}$ is the vacuum state, it will always fuse back into the vacuum. Therefore, if $\gamma$ is a nontrivial representation, then $F_{I \rightarrow \gamma}=0$ and the above operation projects out the $|0, \ldots, 0\rangle$ state.

At this point we have almost constructed a probabilistic projection onto $|0, \ldots, 0\rangle^{\perp} \cap \tilde{\Lambda}$. The states outside of $\tilde{\Lambda}$ can be removed using the probabilistic projection onto $\tilde{\Lambda}$, which for the moment we assume can be implemented. Therefore, the desired gate will be complete if the coefficients

$$
\prod_{\phi \in \Phi} F_{\phi(\lambda) \rightarrow \gamma}
$$

are nonzero and equal for every nontrivial $\lambda \in \tilde{\Lambda}$. The requirement of equality is accomplished if the orbits under the functions in $\Phi$, of all nontrivial $\lambda \in \tilde{\Lambda}$, are equal.

More specifically, let $\Phi$ be a set of maps from $\tilde{\Lambda}$ to $\tilde{\Lambda}$ that fix the identity. We say that $\Phi$ is balanced on $\tilde{\Lambda}$ if it satisfies the relation

$$
\mathcal{N}_{\lambda_{1} \rightarrow \lambda^{\prime}}=\mathcal{N}_{\lambda_{2} \rightarrow \lambda^{\prime}} \quad \forall \lambda_{1}, \lambda_{2}, \lambda^{\prime} \in \widetilde{\Lambda}-\{I\},
$$

where $\mathcal{N}_{\lambda \rightarrow \lambda^{\prime}}$ denotes the number of elements $\phi \in \Phi$ such that $\phi(\lambda)=\lambda^{\prime}$. The requirement that $\Phi$ be balanced guarantees that the expressions in Eq. (112) are equal for every $\lambda$. Of course, for the coefficients to be nonzero, we must prove separately that the value of $F_{\lambda \rightarrow \gamma}$ is nonzero for every nontrivial $\lambda \in \tilde{\Lambda}$.

The goal for the rest of this section is, therefore, to find a subgroup $\widetilde{\Lambda}$ of $\widetilde{H}$, an irreducible representation $R$ of $\widetilde{G}$, and a one-dimensional representation $\gamma$ of $\widetilde{G}$ such that (i) the probabilistic projection onto $\tilde{\Lambda}$ can be implemented, (ii) $F_{\lambda \rightarrow \gamma} \neq 0$ for every nontrivial $\lambda \in \tilde{\Lambda}$, and (iii) there exists a set of maps from $\tilde{\Lambda}$ to $\tilde{\Lambda}$ that is balanced on $\tilde{\Lambda}$ and can be expressed as

$$
\phi(g)=\prod_{i} g_{i} g g_{i}^{-1}
$$

for some elements $\left\{g_{i}\right\} \in \widetilde{G}$.

\section{Choosing $\tilde{\Lambda}$}

There are groups, such as $\mathbb{Z}_{3}^{2} \times_{\theta} D_{4}$, for which there is no choice of $R$ and nontrivial $\gamma$ such that $F_{h \rightarrow \gamma} \neq 0$ for all nontrivial $h \in \widetilde{H}$. It is therefore advantageous to choose $\widetilde{\Lambda}$ as small as possible. Furthermore, a small $\tilde{\Lambda}$ will also help when proving the existence of a set of functions balanced on $\tilde{\Lambda}$.

Let $a$ be a nontrivial element of $\widetilde{H}$, and consider the set of functions of the form

$$
\phi(g)=\prod_{i} g_{i} g g_{i}^{-1},
$$

such that $\phi(a)=I$. The kernel of each of these functions is a subgroup of $\widetilde{H}$ that contains the element $a$. We define $\widetilde{\Lambda}$ as the intersection of all these kernels.

Because there are a finite set of maps from $\widetilde{H}$ to $\widetilde{H}$, we can find a finite set of functions $\left\{\phi_{i}\right\}$, in the form of Eq. (115), satisfying

$$
\begin{gathered}
\lambda \in \widetilde{\Lambda} \Rightarrow \forall i \quad \phi_{i}(\lambda)=I, \\
h \notin \widetilde{\Lambda} \Rightarrow \exists i \quad \phi_{i}(h) \neq I .
\end{gathered}
$$

A probabilistic projection onto $\tilde{\Lambda}$ can be constructed using controlled conjugations on an ancilla $|b\rangle$,

$$
\begin{aligned}
\sum_{h \in \widetilde{H}} \alpha_{h}\left|h b h^{-1}\right\rangle \otimes|b\rangle \rightarrow & \sum_{h \in \widetilde{H}} \alpha_{h}\left|h b h^{-1}\right\rangle \\
& \otimes\left|\phi_{i}(h) b \phi_{i}(h)^{-1}\right\rangle,
\end{aligned}
$$

and then using fusion to make sure that the ancilla remains in the $|b\rangle$ state. Repeating the procedure for each $\phi_{i}$ produces the desired projection.

To build the set of functions that are balanced on $\widetilde{\Lambda}$, let $\Phi$ be the set of functions in the form of Eq. (115) such that $\phi(a) \in \tilde{\Lambda}-\{I\}$. We shall prove that this is the desired set of functions.

Let $\lambda \in \tilde{\Lambda}$ be nontrivial and let $\phi$ be any map in $\Phi$. The value of $\phi(\lambda)$ must be nontrivial and contained in $\tilde{\Lambda}$. Otherwise, it would be possible to construct a map in product form such that $a$ is in its kernel but $\lambda$ is not, contrary to the definition of $\tilde{\Lambda}$. In fact, the functions in $\Phi$ are just automorphisms of $\tilde{\Lambda}$ and form a group with multiplication given by function composition. Furthermore, because $\mathcal{C}_{\widetilde{G}}^{\#}(a)=\widetilde{H}$, for any nontrivial $\lambda \in \tilde{\Lambda}$ there exists a function $\phi_{a \rightarrow \lambda} \in \Phi$ such that $\phi_{a \rightarrow \lambda}(a)=\lambda$. If $\lambda^{\prime} \in \tilde{\Lambda}$ is a third nontrivial element, then for every function $\phi \in \Phi$ such that $\phi(\lambda)=\lambda^{\prime}$ there is a function $\phi^{\prime}(a)=\lambda^{\prime}$ given by $\phi^{\prime}=\phi^{\circ} \phi_{a \rightarrow \lambda}$. Therefore, $\Phi$ is balanced on $\tilde{\Lambda}$.

\section{The amplitudes $\boldsymbol{F}_{h \rightarrow \gamma}$}

To choose $R$ and $\gamma$ we first need to examine and define $F_{h \rightarrow \gamma}$ more carefully. Since we are mostly interested in 
whether $F_{h \rightarrow \gamma}$ is zero or nonzero, we will generally work with its magnitude squared, which has the simple expression

$$
\left.\left|F_{h \rightarrow \gamma}\right|^{2}=\left|P_{\gamma}\right| R(h)\right\rangle\left._{R}\right|^{2},
$$

where $P_{\gamma}$ is the projector onto the space that will turn into the representation $\gamma$ after fusion. This subspace is just the subspace that transforms as $\gamma$ under conjugation. It can be projected out using the orthogonality of characters (and matrix entries for non-Abelian representations):

$$
P_{\gamma}|\Psi\rangle=\frac{1}{|\widetilde{G}|} \sum_{g \in \widetilde{G}} \bar{\gamma}^{g} U(g) \otimes U(g)|\Psi\rangle,
$$

where $\bar{\gamma}$ is the conjugate representation. Note that the values of the representation $\gamma$ on $g \in \widetilde{G}$ will be denoted by $\gamma^{g}$, as a reminder that it is always a power of some root of unity which we shall also denote by $\gamma$.

Combining the expressions for the projector and the electric charge state we obtain

$$
\begin{aligned}
\left|F_{h \rightarrow \gamma}\right|^{2} & \left.=\left|\frac{1}{|\widetilde{G}|} \sum_{g \in \widetilde{G}} \bar{\gamma}^{g}\right| R\left(g h g^{-1}\right)\right\rangle\left._{R}\right|^{2} \\
& =\frac{1}{d_{R}|\widetilde{G}|^{2}}\left|\sum_{g \in \widetilde{G}} \bar{\gamma}^{g} R\left(g h g^{-1}\right)\right|^{2},
\end{aligned}
$$

where $d_{R}$ is the dimension of representation $R$. In the second line, the magnitude squared of the matrix is given by $|M|^{2}$ $=\operatorname{Tr}\left(M M^{\dagger}\right)$, which is equivalent to the sum of the magnitude squared of the entries of the matrix.

Because $\widetilde{H}$ is Abelian, the representation $R$ can be diagonalized on $\widetilde{H}$ so that the diagonal entries are one-dimensional representations of $\widetilde{H}$. These representations can be labeled by an index $i$ running along the diagonal of the matrices $R$ and described by functions $\omega_{i}^{h}: \widetilde{H} \rightarrow \mathrm{C}$. With the new notation,

$$
\left|F_{h \rightarrow \gamma}\right|^{2}=\frac{1}{d_{R}|\widetilde{G}|^{2}} \sum_{i=1}^{d_{R}}\left|\sum_{g \in \widetilde{G}} \bar{\gamma}^{g} \omega_{i}^{g h g^{-1}}\right|^{2},
$$

where the representation $R$ is now implicit in the definition of the representations $\left\{\omega_{i}\right\}$.

Finally, let $\widetilde{S}$ be the stabilizer of $\widetilde{H}$ in $\widetilde{G}$-that is, the subgroup of $G$ that commutes with every element of $\widetilde{H}$. Clearly, it is a normal subgroup of $\widetilde{G}$ and $\widetilde{H} \subset \widetilde{S}$. Furthermore, we had argued that if $q_{i}=p$, then $K_{q_{i}} \in \widetilde{S}$. Therefore $|\widetilde{G} / \widetilde{S}|$ is not divisible by $p$.

Since the function $F_{h \rightarrow \chi}$ will be zero unless we choose a representation such that $\gamma^{S}=1$, we shall assume this from now on and write

$$
\left|F_{h \rightarrow \gamma}\right|^{2}=\frac{|\widetilde{S}|^{2}}{d_{R}|\widetilde{G}|^{2}} \sum_{i=1}^{d_{R}}\left|\sum_{g \in \widetilde{G} / \widetilde{S}} \bar{\gamma}^{g} \omega_{i}^{g h g^{-1}}\right|^{2} .
$$

We are now guaranteed that $\gamma$ corresponds to powers of an $n$th root of unity such that $p$ does not divide $n$. The terms in the above expression have the form

$$
\sum_{i=0}^{p-1} c_{i} \omega^{i},
$$

where the coefficients $c_{i}$ are sums of $n$th roots of unity. By Ref. [7], the expression will be zero if and only if the $n$ coefficients $c_{i}$ are all equal.

Using the above notation it is easy to show two properties of the amplitudes $F_{h \rightarrow \gamma}$. If $\left|F_{h \rightarrow \gamma}\right| \neq 0$, then

$$
\left|F_{h^{j} \rightarrow \gamma}\right|^{2}=\frac{|\widetilde{S}|^{2}}{d_{R}|\widetilde{G}|^{2}} \sum_{i=1}^{d_{R}}\left|\sum_{g \in \widetilde{G} / \widetilde{S}} \bar{\gamma}^{g}\left(\omega_{i}^{g h g^{-1}}\right)^{j}\right|^{2} \neq 0,
$$

as long as $p$ does not divide $j$. Note that in general $\left|F_{h \rightarrow \gamma}\right|$ $\neq\left|F_{h^{j} \rightarrow \gamma}\right|$. The fact that was used above is that $\left|F_{h \rightarrow \gamma}\right| \neq 0$ implies that at least two coefficients of different powers of $\omega$ must be different. Replacing $\omega$ by a power of itself just permutes the coefficients $c_{i}$ in Eq. (123).

The second property is easier to prove in the form of Eq. (121) and states that given $\left|F_{h \rightarrow \gamma}\right| \neq 0$, then

$$
\begin{aligned}
\left|F_{x h x \rightarrow \gamma}\right|^{2} & =\frac{1}{d_{R}|\widetilde{G}|^{2}} \sum_{i=1}^{d_{R}}\left|\sum_{g \in \widetilde{G}} \bar{\gamma}^{g} \omega_{i}^{g x h x g^{-1}}\right|^{2} \\
& =\frac{1}{d_{R}|\widetilde{G}|^{2}} \sum_{i=1}^{d_{R}}\left|\sum_{g \in \widetilde{G}} \bar{\gamma}^{g x^{-1}} \omega_{i}^{g h g^{-1}}\right|^{2}=\left|F_{h \rightarrow \gamma}\right|^{2} \neq 0
\end{aligned}
$$

for any $x \in \widetilde{G}$. The second line involves a relabeling of the summation variable, whereas the third line is true because $\gamma$ is a group homomorphism and $\bar{\gamma}^{-x}$ is just an overall phase.

Together, the two properties imply that, if $\left|F_{h \rightarrow \gamma}\right|^{2}$ is nonzero, then so are the amplitudes $\left|F_{h^{\prime} \rightarrow \gamma}\right|^{2}$ for any nontrivial $h^{\prime}=g h^{i} g^{-1}$. Unfortunately, even after adding the identity element, this set is in general not a group. Furthermore, it remains to be shown that the amplitude is nonzero for at least one $h$.

\section{Finding a nonzero amplitude}

It is possible to indirectly show that, for every element $h \in \widetilde{H}$, there is a pair of representations $R$ and $\gamma$ meeting our requirements, such that $\left|F_{h \rightarrow \gamma}\right|^{2} \neq 0$.

The basic idea is to consider the regular representation of $\widetilde{G}$. Let $\mathcal{H}_{\tilde{G}}$ be the Hilbert space spanned by the vectors

$$
|g\rangle_{\widetilde{G}}
$$

for $g \in \widetilde{G}$. For the moment, these are just abstract vectors in a Hilbert space, and therefore we use the above notation to distinguish them from the anyon magnetic charges.

The group $\widetilde{G}$ has both a left and a right action on this vector space, which transforms as the regular representation 
in both cases. More generally, we could say that there is an action of the group $\widetilde{G} \times \widetilde{G}$ on this vector space given by

$$
|g\rangle_{\widetilde{G}} \rightarrow\left|g_{1} g g_{2}^{-1}\right\rangle_{\widetilde{G}}
$$

for any element $g_{1} \times g_{2} \in \widetilde{G} \times \widetilde{G}$.

Let $\mathcal{H}_{R}$ be the Hilbert space spanned by the vectors of the form $|M\rangle_{R}$, where $R$ in an irreducible representation of $\widetilde{G}$. These spaces are also representations of $\widetilde{G} \times \widetilde{G}$ and, in fact, are irreducible. The space $\mathcal{H}_{\widetilde{G}}$ decomposes as a sum of irreducible representations of $\widetilde{G} \times \widetilde{G}$ as

$$
\mathcal{H}_{\tilde{G}}=\underset{R}{\oplus} \mathcal{H}_{R},
$$

with each irreducible representation $R$ appearing exactly once. Fusion corresponds to a further decomposition into the irreducible representations of the diagonal group $\widetilde{G}$. Therefore, if the state $|h\rangle_{\tilde{G}}$ has a nonzero projection to a representation $\gamma$ of the diagonal group, we know that $\left|F_{h \rightarrow \gamma}\right| \neq 0$ for at least one irreducible representation $R$.

More explicitly, the projection is

$$
P_{\gamma}|h\rangle_{\widetilde{G}}=\frac{1}{|\widetilde{G}|} \sum_{g \in \widetilde{G}} \bar{\gamma}^{g}\left|g h g^{-1}\right\rangle_{\tilde{G}} .
$$

To make it nonzero, it is sufficient to choose $\gamma$ to be constant over the stabilizer, $S_{h}$, in $\widetilde{G}$ of $h$. This is still possible, even with our requirements that $\gamma$ be one dimensional and nontrivial, because $S_{h} / \widetilde{H}$ is a proper subgroup of the nilpotent group $\widetilde{G} / \widetilde{H}$. Proper subgroups of nilpotent groups are always contained in proper normal subgroups because the normalizer of the proper subgroup is always a larger group (and eventually the operation of replacing a subgroup with its normalizer must yield a normal subgroup). This concludes the proof that, for any nontrivial $h \in \widetilde{H}$, there exists a choice of $\gamma$ and $R$ such that $\left|F_{h \rightarrow \gamma}\right| \neq 0$.

In fact, for any two nontrivial elements $\lambda_{1}, \lambda_{2} \in \tilde{\Lambda}$, the same representation $\gamma$ is useful because $S_{\lambda_{1}}=S_{\lambda_{2}}$. However, it is not clear that it is possible to pick $R$ such that both $\left|F_{\lambda_{1} \rightarrow \gamma}\right| \neq 0$ and $\left|F_{\lambda_{2} \rightarrow \gamma}\right| \neq 0$. This is illustrated by working with the group $\mathbb{Z}_{5}^{2} \times{ }_{\theta}\left(\mathbb{Z}_{2} \times \mathbb{Z}_{3}\right)$, where certain choices of $\gamma$ consistent with the above discussion lead to zero amplitudes for at least one nontrivial element of $\widetilde{\Lambda}$, no matter which $R$ is used. On the other hand, the same example does have simultaneous choices of $R$ and $\gamma$ that satisfy all our requirements. It is unclear to the author whether it is possible, for any group $\widetilde{G}$, to choose $R$ and $\gamma$ such that $\left|F_{\lambda \rightarrow \gamma}\right| \neq 0$ for all nontrivial elements $\lambda \in \tilde{\Lambda}$ simultaneously.

\section{Alternative $\tilde{\Lambda}$}

What happens if $R$ and $\gamma$ cannot be chosen so that $\left|F_{\lambda \rightarrow \gamma}\right| \neq 0$ over all nontrivial elements $\lambda \in \tilde{\Lambda}$ ? While none of the examples in this paper have this problem, if such a case arises, we could try to shrink $\tilde{\Lambda}$. In particular, if
$\tilde{\Lambda}=Z_{p}$, then the problem is solved. That is, because we can always choose the representations so that the amplitude is nonzero for some element, then it is guaranteed to be nonzero for the powers of that element as well.

The set of functions balanced on $\widetilde{\Lambda}=\mathbb{Z}_{p}$ can be easily constructed as simply $\phi(g)=g^{i}$ for $0<i<p$. However, the probabilistic projection onto $\tilde{\Lambda}$ is more difficult. It can be achieved if we are willing to relax the error model of the probabilistic projections. That is, we use an approximate probabilistic projection, where the probabilities and projected states are close to the desired results. While the results will be exponentially close in the number of successful fusions, they will only be polynomially close in the number of actual fusions, and therefore the machinery of fault tolerant quantum computation must be employed. Computation with the approximate gate will still be feasible, but one of the advantages of topological quantum computation - that is, the exactness of gates-will be lost.

To construct this approximate projection, consider the amplitude for the fusion of the electric charges into the vacuum, denoted by $F_{h \rightarrow I}$. It is the same quantity that has been dealt with thus far, only with the representation $\gamma$ replaced by the identity representation. These quantities have the expression

$$
\begin{aligned}
\left|F_{h \rightarrow I}\right|^{2} & =\frac{1}{d_{R}|\widetilde{G}|^{2}} \sum_{i=1}^{d_{R}}\left|\sum_{g \in \widetilde{G}} \omega_{i}^{g h g^{-1}}\right|^{2} \\
& =\frac{1}{d_{R}\left|\mathcal{C}_{\widetilde{G}}(h)\right|^{2}} \sum_{i=1}^{d_{R}}\left|\sum_{h^{\prime} \in \mathcal{C}_{\widetilde{G}}(h)} \omega_{i}^{h^{\prime}}\right|^{2},
\end{aligned}
$$

where $\mathcal{C}_{\widetilde{G}}(h)$ is the conjugacy class of $h$ in $\widetilde{G}$. The amplitudes satisfy the properties

$$
0<\left|F_{h \rightarrow I}\right|^{2}<\left|F_{I \rightarrow I}\right|^{2}
$$

for any nontrivial $h \in \widetilde{H}$. The first inequality comes from the fact that we are summing $p$ th roots of unity and the number of summands is not divisible by $p$. The second inequality comes from the fact that $\omega_{i}^{h^{\prime}}$ must be nonconstant over the conjugacy class of $h$. The equation

$$
I=\prod_{h^{\prime} \in \mathcal{C}_{\widetilde{G}}(h)} h^{\prime}
$$

is true because the right-hand side commutes with all of $\widetilde{G}$ and therefore must be the identity. Because the number of factors on the right is not divisible by $p, \omega_{i}$ cannot be constant over the conjugacy class unless it is the identity. Furthermore, since the conjugacy class generates $\widetilde{H}$ and $R$ is nontrivial, one of the $\omega_{i}$ must not be the identity. This proves the second inequality of Eq. (131).

The standard procedure of entangling a state with an electric charge pair, which is then fused, can then be used. The state is now kept if the pair fuses into the vacuum, which always has a nonzero probability of occurring. The basis state that was entangled with $|R(I)\rangle_{R}$ will have its amplitude increased relative to the other basis states. Using braiding to achieve a function of the form $f(h)=h a^{i}$, for some element $a \in \tilde{\Lambda}$ and different values of $i$, we can make the basis states 
in $\tilde{\Lambda}$ consisting of powers of $a$ have an arbitrarily large amplitude relative to the other states. Even if $\left|F_{h \rightarrow I}\right|$ varies significantly over the nontrivial elements of $\widetilde{H}$, we can use the old $\widetilde{\Lambda}$ projector and functions in $\Phi$ to balance out the nontrivial elements while increasing the amplitude of the state with $f(h)=I$. After many repetitions, the basis states with flux $a^{i} b a^{-i}$ can be made to have an amplitude much larger then all the other states. This completes the construction of the approximate probabilistic projection onto the new $\tilde{\Lambda}$ for the special cases when we require $\widetilde{\Lambda}=Z_{p}$.

\section{G. Putting it all together}

At this point we have shown the existence of an extended computational space, with elements labeled by $\widetilde{H}=Z_{p}^{n}$, on which we can perform the generalized controlled- $X$ gate, and probabilistic projections onto $|0, \ldots, 0\rangle$ and $|\widetilde{0}, \ldots, \widetilde{0}\rangle$. Furthermore, there exists a nontrivial subgroup $\widetilde{\Lambda} \subset \widetilde{H}$, such that we can implement probabilistic projections onto $\tilde{\Lambda}$ and $|0, \ldots, 0\rangle^{\perp} \cap \tilde{\Lambda}$.

To define the real computational subspace, choose a nontrivial element $a \in \tilde{\Lambda}$ and define

$$
|i\rangle \equiv\left|a^{i} b a^{-i}\right\rangle,
$$

for $0 \leqslant i<p$. This subspace corresponds to the subgroup $\left\{a^{i}\right\} \subset \tilde{\Lambda}$ of powers of $a$.

A probabilistic projection onto the real computational space, corresponding to $\left\{a^{i}\right\}$, can be achieved in two steps. The first step is to apply the probabilistic projection onto $\widetilde{\Lambda}$. The second step is repeated for each $\lambda \in \tilde{\Lambda}$ that is not in $\left\{a^{i}\right\}$. For fixed $\lambda$, we use an ancilla to conjugate by $\lambda^{-1}$, then do the probabilistic projection onto $|0, \ldots, 0\rangle^{\perp} \cap \tilde{\Lambda}$, and then conjugate by $\lambda$ using another ancilla:

$$
\begin{aligned}
\sum_{x \in \tilde{\Lambda}} \alpha_{x}\left|x b x^{-1}\right\rangle & \rightarrow \sum_{x \in \tilde{\Lambda}} \alpha_{x}\left|\lambda x b x^{-1} \lambda^{-1}\right\rangle \\
& \rightarrow C \sum_{x \in \tilde{\Lambda}, x \neq \lambda} \alpha_{x}\left|\lambda x b x^{-1} \lambda^{-1}\right\rangle \\
& \rightarrow C \sum_{x \in \tilde{\Lambda}, x \neq \lambda} \alpha_{x}\left|x b x^{-1}\right\rangle,
\end{aligned}
$$

where the probabilistic projection was assumed to succeed in the second step, and therefore the state is renormalized by the constant $C$. The net effect of one such operation is to project out the state $\left|\lambda b \lambda^{-1}\right\rangle$. If all the projections succeed, then we will have projected the original state into the computational basis, completing the probabilistic projection onto $\left\{a^{i}\right\}$.

For the case of qudits with $d=p>2$ we are now done. The generalized controlled- $X$ gate behaves as a controlled- $X$ gate when restricted to act on the computational space. A probabilistic projection onto $|0\rangle$ is just the probabilistic pro- jection onto $|0, \ldots, 0\rangle$ because $|0, \ldots, 0\rangle=|0\rangle$. The probabilistic projection onto $|\widetilde{0}, \ldots, \widetilde{0}\rangle$ behaves as a probabilistic projection onto $|\widetilde{0}\rangle$ because

$$
\langle\widetilde{i} \mid \widetilde{0}, \ldots, \widetilde{0}\rangle \propto \delta_{i, 0},
$$

with the caveat that we must use the projection onto the computational basis to turn the $|\widetilde{0}, \ldots, \widetilde{0}\rangle$ ancillas into $|\widetilde{0}\rangle$ ancillas. Finally, the probabilistic projection onto $|0, \ldots, 0\rangle^{\perp} \cap \tilde{\Lambda}$ reduces to a probabilistic projection onto $|0\rangle^{\perp}$ when acting on states in the computational subspace. These are the gates that were proven universal for quantum computation in Sec. IV.

\section{Case $p=2$}

Special treatment must be given to the case when $p=2$ - that is, when working with qubits. Though all the gates constructed above are valid for $p=2$, the gate set is not universal. The problem is that the probabilistic projection onto $|0\rangle^{\perp}=|1\rangle$ does not provide any additional computational power beyond the probabilistic projection onto $|0\rangle$.

Just as in Sec. IV B, the gate set can be made universal given a supply of the magic states:

$$
\begin{gathered}
\left|\phi_{M 1}\right\rangle=\frac{1}{2} \sum_{i, j}|i\rangle \otimes|j\rangle \otimes|i j\rangle, \\
\left|\phi_{M 2}\right\rangle=\frac{1}{2} \sum_{i, j} \omega^{\delta_{i, 1} \delta_{j, 1}|i\rangle} \otimes|j\rangle,
\end{gathered}
$$

where the second state can be produced from the first one by measuring the third qudit in the $X$ basis.

The production of the magic state $\left|\phi_{M 1}\right\rangle$ is the step that requires a projection constructed from the fusion of electric charges. Given our choice of $a \in \tilde{\Lambda}$ above, assume that $b a b^{-1} \in \tilde{\Lambda}$. This must be the case if $\tilde{\Lambda}$ was defined as the intersection of kernels of functions. Clearly, we can apply a controlled conjugation by $a$ and therefore, additionally, the controlled conjugation by $b a b^{-1}$ and by $a b a b^{-1}$. Note that $a \neq b a b^{-1}$ because $b$ was chosen to not commute with $a$.

We begin with the $|\widetilde{0}\rangle \otimes|\widetilde{0}\rangle \otimes|\widetilde{0}\rangle$ state and append a $|0\rangle$ $=|b\rangle$ ancilla. We then conjugate it to obtain

$$
\frac{1}{\sqrt{8}} \sum_{i=0}^{1} \sum_{j=0}^{1} \sum_{k=0}^{1}|i\rangle \otimes|j\rangle \otimes|k\rangle \otimes\left|f_{i, j, k} b f_{i, j, k}^{-1}\right\rangle,
$$

where

$$
f_{i, j, k}=a^{1-i}\left(b a b^{-1}\right)^{1-j} x^{k},
$$

with $x$ to be determined in a moment. A probabilistic projection onto $|0, \ldots, 0\rangle^{\perp}$ is then applied to the last ancilla, and the conjugations are undone.

If the projection succeeds, we will have projected out two out of the initial eight basis states, depending on the value of $x \in\left\{a, b a b^{-1}, a b a b^{-1}\right\}$. In all cases, the state $|1\rangle \otimes|1\rangle$ $\otimes|0\rangle$ is removed, and for each of the three values of $x$, one 
of the other undesirable basis states is removed. Repeating the procedure once for each value of $x$ produces the desired magic state $\left|\phi_{M 1}\right\rangle$. Note that the above procedure succeeds because $a^{2}=1$ and $a$ commutes with $b a b^{-1}$.

What happens if $b a b^{-1}$ is not in $\tilde{\Lambda}$ ? This is the case when fusions of electric charges into the vacuum must be used. In particular, instead of projecting out the undesirable basis states, we increase the amplitude of the desired basis states and obtain an ancilla that is exponentially close to the desired magic state. The procedure is almost unchanged, except that the function involved is

$$
f_{i, j, k}=a^{i}\left(b a b^{-1}\right)^{j} x^{1-k},
$$

and the function $f_{i, j, k}^{\prime}=a^{i}\left(b a b^{-1}\right)^{j}$ must also be used to adjust the relative amplitude of $|1\rangle \otimes|1\rangle \otimes|1\rangle$ with respect to the other desired states.

In either case, we have now shown that case of qubits can be dealt with in a similar fashion to the general qudit case, and therefore, we have completed the construction of universal quantum computation for anyons based on solvable nonnilpotent groups.

\section{LEAKAGE CORRECTION}

Before concluding this paper, it is important to address the issue of fault tolerance. A physical system with anyons will have sources of errors due to the finite separation of anyons and nonzero temperature (see Refs. $[1,3,4]$ for details). While the probability of error is exponentially small in the distance and temperature, it is in general nonzero. These errors could be especially relevant if anyons are used as long-term quantum memory, in which case error correcting codes must be employed.

While most of the machinery of error correcting codes can be applied directly to anyons, it requires that states with errors remain within the computational subspace (that is, the subspace on which universal quantum computation can be done). For our model of computation, this is only a small subspace corresponding to anyons that are magnetic charges with fluxes such as $a^{i} b a^{-i}$ and arranged in pairs of trivial total flux. Note that only the magnetic charges need error correction as they are the ones in which the quantum state is stored.

All that is required to perform quantum error correction is to be able to replace qudits that have "leaked out" of the computational subspace with arbitrary states that are in the computational subspace. This step can then be followed by the standard error correcting step, which will remove the errors. The leakage correction step is equivalent to the swapif-leaked gate described by Kempe et al. [12].

In Ref. [1] a leakage correction scheme was presented for nonsolvable anyons. While a similar scheme could be constructed for the solvable anyons discussed in the present paper, it will be easier to present a generic leakage correction scheme that can also be applied to anyons.

The scheme is simply to teleport a computational qudit to a fresh qudit. The standard steps, shown in Fig. 1, are first to create the entangled ancilla $|\Phi\rangle=\Sigma_{i}|i\rangle \otimes|i\rangle / \sqrt{d}$ and then

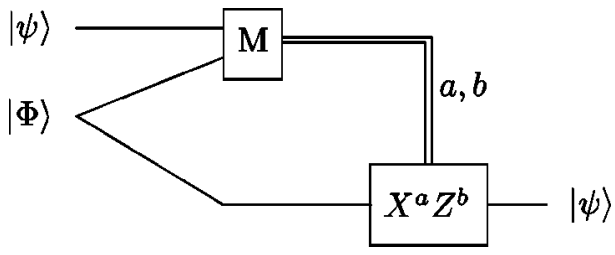

FIG. 1. Leakage correction circuit.

measure the computational qudit and the first ancilla qudit in the basis $|a, b\rangle=X^{a} Z^{b} \otimes I|\Phi\rangle$, obtaining outcome $a, b$. The correction gate $X^{a} Z^{b}$ is then applied to the second ancilla qudit, which now becomes part of the computational space. All these operations can be performed using the anyon gates discussed so far.

If the original qudit was in the computational space, then its state will be flawlessly transfered into the new qudit (in our case, a fresh anyon pair). However, if the original qudit had leaked, then the new qudit will be guaranteed to be in the computational subspace, because it was obtained by applying Pauli operators to a qudit known to be in the computational subspace. This is the desired leakage correction protocol.

In fact, this scheme can be applied to almost any system, as long as we can guarantee that the measurement of the first two qudits will not affect the third qudit in any way, as should be the case if they are sufficiently separated.

The leakage correction scheme has caveat from a theoretical standpoint, though. We are effectively assuming that we possess a classical leakage detection machine, through which the data " $a, b$ " is run. That is, if the measurement produced an outcome in the form of a voltage and then the gate $X^{a} Z^{b}$ was constructed as a Hamiltonian controlled by this voltage, we would need to guarantee that only the $d^{2}$ acceptable voltage signals could reach the machine operating on the third qudit. However, in practice, leakage correcting a classical signal is trivial, as classical information can be measured without any negative side effects.

A very similar scheme can be produced given a quantum system that is known to have exactly $d$ states. The qudit is simply swapped into the new system; the first system is then erased and restored into the computational space, and then the qudit is swapped back. In this context, the teleportation scheme is in effect a way of swapping a qudit into a classical system.

Though the leakage correction scheme was discussed in general terms, it clearly applies to the anyons discussed in this paper, and its use allows quantum error correction and fault tolerance to be employed. We have therefore shown that even in the presence of small sources of noise, the anyons can still be used for universal quantum computation.

\section{CONCLUDING REMARKS}

The main result of this paper is that anyons from finite groups that are solvable but not nilpotent are capable of universal quantum computation. This set includes many groups of small size, which are more likely to be found in a physical system. Combined with the results of Ref. [1], we have proved that every finite group that is not nilpotent produces 
anyons capable of universal quantum computation.

Furthermore, except for the groups where the methods of Sec. VI F 4 must be used, the computations with anyons can be made error free in the following sense: in the theoretical limit of zero temperature and infinite separation between anyons, an arbitrarily long calculation can proceed without the need of error correction. The elementary unitaries are always perfect, whereas the measurements are either perfect or are known to have failed (i.e., when none of the probabilistic projections succeed). This occurs with a probability that can be made exponentially small in the number of fusions. Of course, a real system will have additional exponentially small errors due to finite size and temperature effects.

The physical requirements for the constructions in this paper include a supply of electric charge ancillas, in addition to the requirements of Ref. [1]. The necessity of the electric charges may present an extra source of difficulties for a real implementation. The exception is $S_{3}$, in which case only magnetic charges are required, as mentioned at the end of Sec. III. In either case, the issue of producing the elementary electric or magnetic ancillas is not addressed in this paper, though a generalization of the construction in Ref. [1] may be sufficient.

Another open question is whether anyons from nonAbelian nilpotent groups are capable of universal quantum computation. Additionally, not much is known about computing with anyons that do not belong to the electric and magnetic charge model discussed in this paper. On the other hand, the universality of anyons from certain continuous groups has been discussed in Refs. [13,14].

Of course, the most important open question is whether we can find a laboratory system with anyons out of which a quantum computer can be built. The requirement of a twodimensional space severely limits the possibilities. However, certain exotic systems such as the fractional levels of the quantum Hall effect may contain non-Abelian anyons. Another option is the possibility of engineering a system with the desired anyons. Recent proposals include using optical lattices [15] or Josephson-junction arrays [16]. In the latter case, an explicit array is constructed that simulates $S_{3}$ gauge theory on a lattice. Ideally, one day such a system could be used to turn the ideas presented here into a working quantum computer.

\section{ACKNOWLEDGMENTS}

Much of this work is inspired by the construction of Alexei Kitaev, who showed that universal computation was possible with anyons based on the group $S_{3}$. His construction used the magic state $(1 / \sqrt{3})(|0\rangle-|1\rangle-|2\rangle)$ to build a qubit Toffoli gate. Many of the above ideas were captured by the unpublished notes of John Preskill. I am also indebted to Charlene Ahn and Ben Toner who were kind enough to read and review this paper. This work was supported in part by the National Science Foundation under Grant No. EIA0086038 and by the U.S. Department of Energy under Grant No. DE-FG03-92-ER40701.
[1] C. Mochon, Phys. Rev. A 67, 022315 (2003).

[2] A.Yu. Kitaev, Ann. Phys. (N.Y.) 303, 2 (2003).

[3] R.W. Ogburn and J. Preskill, in QCQC '98, edited by C.P. Williams (Springer-Verlag, Berlin, 1999), pp. 341-356.

[4] J. Preskill, e-print quant-ph/9712048.

[5] F.A. Bais, P. van Driel, and M. de Wild Propitius, Phys. Lett. B 280, 63 (1992).

[6] M. de Wild Propitius and F.A. Bais, e-print hep-th/9511201.

[7] I.J. Schoenberg, Mathematika 11, 131 (1964).

[8] D. Gottesman, in QCQC '98 (Ref. [3]), pp. 302-313.

[9] P.W. Shor, in 37th Symposium on Foundations of Computer Science (IEEE Computer Society Press, Los Alamitos, CA,
1996), pp. 56-65.

[10] D.A. Barrington, J. Comput. Syst. Sci. 38, 150 (1989).

[11] D.A.M. Barrington, H. Straubing, and D. Therien, Inf. Comput. 89, 109 (1990).

[12] J. Kempe, D. Bacon, D.P. DiVincenzo, and K.B. Whaley, Quantum Inf. Comput. 1, 33 (2001).

[13] M. Freedman, Found. Comput. Math. 1, 183 (2001).

[14] M. Freedman, A. Kitaev, M. Larsen, and Z. Wang, Bull. Am. Math. Soc. 40, 31 (2003).

[15] L.-M. Duan, E. Demler, and M.D. Lukin, Phys. Rev. Lett. 91, 090402 (2003).

[16] B. Doucot, L.B. Ioffe, and J. Vidal, e-print cond-mat/0302104. 